\title{
Asymptotic Expansion for the Density of States of the Magnetic Schrödinger Operator with a Random Potential
}

\section{Wei-Min Wang}

UA 760 du CNRS, Département de Mathématiques, Université Paris-Sud, Bâtiment 425, F-91405 Orsay Cedex, France

Received: 1 June 1994/in revised form: 13 December 1994

\begin{abstract}
We study the asymptotics for the density of states of the magnetic Schrödinger operator with a random potential. By using the methods of effective Hamiltonian, complex dilation and complex translation, we obtain in the large magnetic field limit, the asymptotic expansion for the density of states measure considered as a distribution.
\end{abstract}

\section{Introduction}

We study the density of states of the magnetic Schrödinger operator with a random potential defined on $L^{2}\left(\mathbb{R}^{2}\right)$

$$
P_{B, V}^{(1)}=\left(D_{x}+B y\right)^{2}+D_{y}^{2}+V^{(1)}(x, y),
$$

where $D_{x}=(1 / i) \partial_{x}, D_{y}=(1 / i) \partial_{y}$ and $B>0$ is a constant. Let $v$ be a $C_{0}^{\infty}$ function, the potential $V$ is defined as

$$
V(\bar{x})=\sum_{i \in \mathbb{Z}^{2}} \alpha_{i} v(\bar{x}-i)=\sum_{i \in \mathbb{Z}^{2}} \alpha_{i} v_{i}(\bar{x})
$$

where $\bar{x}=(x, y), \alpha=\left\{\alpha_{i}\right\}_{i \in \mathbb{Z}^{2}}$ form a random field, i.e. a family of random variables indexed by $\mathbb{Z}^{2}$ on a probability space $(\Omega, P)$. We denote by $\langle f\rangle$ the expectation value of the random variable $f$. One can always suppose that $\Omega=\mathbb{R}^{\mathbb{Z}^{2}}$. In this case,

$$
\alpha_{(j)}(j)=\omega(j),
$$

and the translation operators, $T_{i}\left(i \in \mathbb{Z}^{2}\right)$ in $\Omega$ are defined by

$$
T_{i} \omega(j)=\omega(j-i) .
$$

We thank Johannes Sjöstrand for many illuminating discussions as well as the Mittag-Leffler Institute for its hospitality while part of this work was done. We also thank Bernard Helffer for his helpful comments on this article. 
Hence for random potentials of the form (1.1), we have, by using (1.2) and (1.3),

We take

$$
V^{T_{l}^{(1)}}(\bar{x})=V^{(1)}(\bar{x}-i) .
$$

$$
d P=\prod_{l \in \mathbb{Z}^{2}} g\left(\alpha_{l}\right) d \alpha_{i},
$$

where $g$ is a $C_{0}^{\infty}$ function. We say that the $\alpha_{l}$ are independent identically distributed (i.i.d.) random variables. The action of $T_{j}$ is ergodic on $(\Omega, P):$ if $A \subset \Omega$ satisfies

$$
\forall j \in \mathbb{Z}^{2}, T_{j}^{-1} A=A,
$$

then $P(A)=0$ or 1 . The operator $P_{B, V}^{()}$is an ergodic operator.

When $V=0$, the Hamiltonian $P_{B, 0}$ has eigenvalues $\lambda_{n}=(2 n+1) B, n \in \mathbb{N}$ with infinite multiplicity. These are the so-called Landau levels.

We define the "magnetic translations" $\tau_{j}^{B}\left(j=\left(j_{1}, j_{2}\right) \in \mathbb{Z}^{2}\right)$ by

In particular

$$
\left[\tau_{j}^{B} u\right](x, y)=e^{-l B j_{2}\left(x-\frac{1}{2} j_{1}\right)} u\left(x-j_{1}, y-j_{2}\right) .
$$

$$
\begin{aligned}
& {\left[\tau_{\left(J_{1}, 0\right)} u\right](x, y)=u\left(x-j_{1}, y\right),} \\
& {\left[\tau_{\left(0, J_{2}\right)} u\right](x, y)=e^{-\imath B J_{2} x} u\left(x, y-j_{2}\right) .}
\end{aligned}
$$

Let $j \wedge k$ be the determinant of $(j, k)$ in the canonical basis. We have the relations

When $V \neq 0$, we have

$$
\begin{aligned}
& {\left[P_{B, 0}, \tau_{j}^{B}\right]=0,} \\
& \tau_{j}^{B} \tau_{k}^{B}=e^{\frac{1}{2} \iota B(j \wedge k)} \tau_{,+k}^{B}, \\
& \tau_{j}^{B} \tau_{k}^{B}=e^{\imath B(\jmath \wedge k)} \tau_{k}^{B} \tau_{,}^{B},
\end{aligned}
$$

$$
\tau_{-,}^{B} P_{B, V}^{(1)} \tau_{J}^{B}=P_{B, V}^{T_{,}(1)}
$$

where we used (1.4). Hence by standard arguments concerning ergodic operators [CFKS, Pa], the spectrum of $P_{B, v}^{(\omega)}, \sigma\left(P_{B, v}^{(1)}\right)$ is almost surely constant with respect to $\omega$, i.e.

$$
\sigma\left(P_{B, v}^{(1)}\right)=\sigma\left(P_{B, v}\right) \text { a.s. , }
$$

where $\sigma\left(P_{B, v}\right)$ is a non-random set. Without loss of generality we may assume range $v \subset[-1,1]$. If moreover we take supp $g \subset(-p, p)$ with $p<B$, then $\sigma\left(P_{B, v}^{(o)}\right)$ is contained in the union $\cup_{n}\left[\lambda_{n}-p, \lambda_{n}+p\right]$. We will show later that the density of states measure $\rho^{(1)}$ is also almost surely non-random:

$$
\rho^{(1)}=\rho \quad \text { a.s. , }
$$

where $\rho$ is a non-random measure.

The density of states of the magnetic Schrödinger operator with a random potential was first studied by the physicist Wegner [We] (see also [BGI, KP]). This is a physically measurable quantity. In the strong field limit $B \gg 1$, Wegner developed a heuristic argument. According to his argument, if one is only interested in the density of states for low energies, one only needs to take into account the lowest Landau level, and one can neglect the contributions of other Landau levels. This way Wegner was able to compute exactly the first term of the density of states for a Gaussian white noise potential. 
In this paper, we justify the Wegner approximation in some weak sense, and obtain an asymptotic expansion for $\rho$ considered as a distribution. We show that, there exist $C^{\infty}$ functions $\rho_{l}(t)$, such that for all $N_{0} \in \mathbb{N}, c>0$, if $f \in$ $C_{0}^{\infty}(((-B,-c) \cup(c, B))+(2 n+1) B)$ satisfies $\left|\partial^{j} f\right|=\mathbf{O}\left(B^{N_{0} \prime}\right)$ for $B \gg 1$, then for every $m \in \mathbb{N}$ :

$$
\begin{aligned}
\int f(E) d \rho_{B}(E)=B & \int f(t+(2 n+1) B) \rho_{0}^{(n)}(t) d t \\
& +\int f(t+(2 n+1) B) \rho_{1}^{(n)}(t) d t \\
& +B^{-1} \int f(t+(2 n+1) B) \rho_{2}^{(n)}(t) d t+\cdots \\
& +B^{-(m-1)} \int f(t+(2 n+1) B) \rho_{m}^{(n)}(t) d t+\mathbf{O}\left(B^{-m}\right),
\end{aligned}
$$

where $t=E-(2 n+1) B$ is the renormalized energy and $d \rho_{B}(E)$ is the "nonrandom" density of states measure that we mentioned earlier. In particular, if $v$ has support contained in the unit square centered at the origin, we have

$$
\begin{gathered}
\rho_{0}^{(n)}(t)=\frac{1}{2 \pi} \int F_{0}(\gamma) g\left(\frac{t}{\gamma}\right) \frac{d \gamma}{|\gamma|}, \\
\text { where } \quad F_{0}(\gamma)=\int_{t=;} \frac{d s}{\|\nabla v\|} ;
\end{gathered}
$$

(note that $\rho_{0}^{(n)}(t)$ is independent of $n$ ) and

$$
\begin{aligned}
& \rho_{1}^{(n)}(t)=\frac{2 n+1}{8 \pi} \int F_{1}(\gamma) g\left(\frac{t}{\gamma}\right) \frac{d \gamma}{|\gamma|}, \\
& \text { where } F_{1}(\gamma)=\frac{d}{d \gamma} \int_{l=;} \frac{\Delta v}{\|\nabla v\|} d s .
\end{aligned}
$$

If we further assume that $v \geqq 0$ and that the supports of $v_{l}$ intersect so that

$$
\sum_{l \in \mathbb{Z}^{2}} v_{l} \geqq s>0
$$

for some postive constant $s$, then we show that the expansion (1.5) holds for all $f \in C_{0}^{\infty}((-B, B)+(2 n+1) B)$ satisfying $\left|\partial^{j} f\right|=\mathbf{O}\left(B^{N_{0 J} J}\right)$.

We use the Grushin method to study this problem as Helffer and Sjöstrand [HS] did in the case where $V$ is periodic. We take $h=B^{-1} \ll 1$ to be our semi-classical parameter. For all $z^{\prime}$ in $\left[\lambda_{n}-a, \lambda_{n}+a\right]$, the study of $P_{B, b}^{(1)}-z^{\prime}$ can be reduced to the study of an effective one-dimensional operator denoted by $E_{-+} / h: z^{\prime} \in \sigma\left(P_{B .1}^{(\prime)}\right)$ if and only if $0 \in \sigma\left(E_{-+} / h\right)$. The symbol of $E_{-+}$has an asymptotic expansion in $h$, the principal symbol being $h(V(x, \xi)-z)$, where $z=z^{\prime}-\lambda_{n}$.

The effective Hamiltonian is the starting point of our analysis. The additional parameters $\alpha_{i}$ and the associated probability density $g\left(\alpha_{i}\right)$ enable us to use scaling arguments (see Sect. IV). To order $\mathbf{O}\left(h^{\infty}\right), E_{-+}^{(1)}(x, \xi)$ only depends on the potential and its derivatives at $(x, \xi)$. For $\left|\operatorname{Im} z^{\prime}\right| \geqq h^{\frac{1}{2}-\varepsilon}(\varepsilon>0),\left(E_{-+}^{(1)}\right)^{-1}$ admits a parametrix, i.e. an asymptotic expansion in powers of $h$, the first term being

$$
\frac{1}{h\left(V^{(1)}-z\right)} .
$$


In order to study the density of states, we need to have control over the non-elliptic regions where $\left|x_{j} v_{j}-z\right| \ll 1$. By the ergodic theorem, the density of states $\rho^{(1)}$ exists almost surely and is equal to the averaged density of states $\rho$ (see Sect. III). Once we take the average, we have

$$
\left\langle E_{-+}^{-1}(x, \xi)\right\rangle=\left\langle E_{-+}^{-1}\left(x+i_{1}, \xi+i_{2}\right)\right\rangle
$$

$\left(\left(i_{1}, i_{2}\right) \in \mathbb{Z}^{2}\right)$. Therefore we only need to have bounds on $\left\langle E_{-+}^{-1}(x, \xi)\right\rangle$ for $(x, \xi) \in \mathbb{E}$ with $\mathbb{E}$ the unit square centered at the origin.

Due to the "local" nature of the operator $E_{-+}(x, \xi)$; the influence of the nonelliptic regions on $E_{-+}^{-1}(x, \xi)$ decreases as $j$ increases. To exhibit this, we complex dilate in $\alpha_{j}: \alpha_{j} \rightarrow \alpha_{j} e^{\imath()_{l}}$, where $\theta_{j} \cong 1 /|j|$.

This is our scaling argument in dimension 2. (The precise form of the scaling relation depends crucially on the dimension.) We can in this way control the non-elliptic regions up to the scale $L=\mathbf{O}\left(h^{-N}\right)$ for all $N \in \mathbb{N}$. Hence for all $z$ such that $\operatorname{Re} z \neq 0$, the asymptotic expansion for $\left\langle E_{-+}^{-1}\right\rangle$ is valid for $|\operatorname{Im} z|$ not too small $\left(|\operatorname{Im} z|^{-1}=\mathbf{O}\left(h^{-N}\right)\right)$. For all fixed $N_{0} \in \mathbb{N}$, we study the integral $\int f(t) d \rho(t)$ for all $f$ such that $\left|\partial^{\prime} f\right|=\mathbf{O}\left(h^{-N_{0} j}\right)$ and with support away from $\{(2 n+1) B, n \in \mathbb{N}\}$. We show that the $\rho_{l}$ are $C^{\infty}$ functions away from 0 .

If we make the further assumption (1.6), then we complex translate in $\alpha_{1}$ : $\alpha_{j} \mapsto \alpha_{j}+i \delta_{j}$, where $\delta_{j}=1 /|j|$, to control the non-elliptic regions. We show that (1.5) holds for all $f$ such that $\left|\partial^{j} f\right|=\mathbf{O}\left(h^{-N_{0} /}\right)$ and that the $\rho_{l}$ are $C^{\infty}$ functions.

By a standard procedure, (1.5) can be extended to include Hölder continuous functions ( $B$ independent) $f \in C^{\alpha}, 0<\alpha<1$ with an error which is of order $\mathbf{O}\left(h^{\infty}\right)$.

This result should be compared with the periodic case:

$$
V(\bar{x})=\sum_{l \in \mathbb{Z}^{2}} v(\bar{x}-i)
$$

i.e. $\alpha_{l}=1$ for all $i \in \mathbb{Z}^{2}$. There one needs much stronger conditions on $f$, namely $\left|\partial^{J} f\right|=\mathbf{O}\left(h^{-j /(2+\varepsilon)}\right)(\varepsilon>0)$. Moreover, in the periodic case, $\rho_{l}$ has singularities. Of course this is due to the fact that our estimates on the parametrix for $E_{-+}^{-1}$ is valid only for $|\operatorname{Im} z| \geqq h^{\frac{1}{2}-\varepsilon}$.

The nature of the spectrum of $P_{B, V}^{(1)}$ is presently not known. The operator $P_{B, V}^{(1)}$ is conjectured to have pure point spectrum with localized eigenfunctions (commonly known as localization) for certain ranges of energies. This conjecture has been used in building theories of the quantum Hall effect [Bel]. It is our eventual aim to prove this conjecture and this paper constitutes a first step toward research in that direction.

\section{The Reduction and the Associated Grushin Problem}

Classes of Symbols. We say that a symbol $Q(x, \xi, h)$ is in the class $S^{0}\left(\mathbb{R}^{2}\right)$ if it verifies

$$
\left.\left.\exists h_{0}, \forall \alpha \in \mathbb{N}^{2}, \exists C_{\alpha}, \forall(x, \xi) \in \mathbb{R}^{2}, \forall h \in\right] 0, h_{0}\right],\left|D_{x}^{\alpha_{1}} D_{\zeta}^{\alpha_{2}} Q(x, \xi, h)\right| \leqq C_{x} .
$$


One can associate with it a pseudo-differential operator (p.d.o.) (this process is called the $h$-quantization of Weyl)

$$
\left(Q^{W}\left(x, h D_{x}, h\right) u\right)(x)=(2 \pi h)^{-1} \int e^{(1 / h)\left\langle x-x^{\prime}, \xi\right\rangle} Q\left(\frac{x+x^{\prime}}{2}, \xi, h\right) u\left(x^{\prime}\right) d x^{\prime} d \xi .
$$

Similarly, one can define the classes of operator valued symbols:

$$
\begin{aligned}
& S^{0}\left(\mathbb{R}_{x, \xi}^{2} ; \mathscr{L}\left(B_{y}^{k}, B_{y}^{k^{\prime}}\right)\right), \\
& S^{0}\left(\mathbb{R}_{r, \zeta}^{2} ; \mathscr{L}\left(B_{y}^{h}, \mathbb{C}\right)\right), \\
& S^{0}\left(\mathbb{R}_{x, \xi}^{2} ; \mathscr{L}\left(\mathbb{C}, B_{y}^{h}\right)\right),
\end{aligned}
$$

where the $B_{y}^{k}(k \in \mathbb{Z})$ are defined by:

$$
\begin{aligned}
& B_{y}^{0}=L^{2}\left(\mathbb{R}_{y}\right), \\
& B_{y}^{k}=\left(1+D_{y}^{2}+y^{2}\right)^{-k} B_{y}^{0} .
\end{aligned}
$$

$B_{y}^{-k}$ is the dual of $B_{y}^{k}$, and $\mathscr{L}(E, F)$ designates the space of bounded operators from $E$ to $F$. Unless specified otherwise, we will in general denote the symbol and its corresponding operator by the same letter. We will use $\#\left(\#_{h}\right)$ to denote the composition of 1-quantized ( $h$-quantized) Weyl symbols.

In this paper, we will also frequently encounter $Q$, symbols in $S^{0}\left(\mathbb{R}_{x, \xi, y, \eta}^{4}\right)$. We associate with $Q$ the following p.d.o. (the Weyl $(h, 1)$-quantization):

$$
\begin{aligned}
& \left(Q^{W}\left(x, h D_{x}, y, D_{y}, h\right) u\right)(x, y) \\
& \quad=\left(4 \pi^{2} h\right)^{-1} \int e^{(i / h)\left\langle x-x^{\prime}, \xi\right\rangle} e^{i\left\langle y-y^{\prime}, \eta\right\rangle} Q\left(\frac{x+x^{\prime}}{2}, \frac{y+y^{\prime}}{2}, \xi, \eta, h\right) u\left(x, y^{\prime}\right) d x^{\prime} d y^{\prime} d \xi d \eta .
\end{aligned}
$$

We identify $Q$ with operator valued symbols in $S^{0}\left(\mathbb{R}_{x, \xi}^{2} ; \mathscr{L}\left(L^{2}\left(\mathbb{R}_{y}\right), L^{2}\left(\mathbb{R}_{y}\right)\right)\right)$. We use \# to denote the composition of such operator valued symbols when the $(x, \xi)$ variables are held fixed and the composition is only in the $(y, \eta)$ variables.

We now consider the operator $P_{B, v}^{(1)}$ in the introduction. By a standard argument, there exists a unitary operator $U$ such that $P_{B, V}^{\omega}$ is unitarily equivalent to

$$
\tilde{P}_{B, V}^{\omega}=U P_{B, V}^{(\omega)} U^{-1}=B\left(D_{y}^{2}+y^{2}\right)+V^{W}\left(x+B^{-1 / 2} y, B^{-1} D_{x}-B^{1 / 2} D_{y}\right) .
$$

(See Proposition 1.10 of [HS]). Renormalizing, we get (for simplicity, we now generally drop the superscript $\omega$ )

$$
H_{B, V}^{(n)}=D_{y}^{2}+y^{2}-(2 n+1)+B^{-1} V^{W}\left(x+B^{-1 / 2} y, B^{-1} D_{x}-B^{-1 / 2} D_{y}\right),
$$

where $n$ is the Landau level that we are interested in, $B^{-1}=h$ is our semi-classical parameter as mentioned earlier. (Note that the symbol of $V^{W}$ is $V\left(x+h^{1 / 2} y\right.$, $\left.\xi-h^{1 / 2} \eta\right)$.)

Let $z$ be a complex parameter such that $h z$ is in the open disc $B(0,1)$ centered at 0 with radius 1 . Define $R_{-}^{(n)}$ to be the operator from $L^{2}\left(\mathbb{R}_{x}\right)$ to $L^{2}\left(\mathbb{R}_{x, y}^{2}\right)$ such that

$$
\left(R_{-}^{(n)} v_{-}\right)(x, y)=h_{n}(y) v_{-}(x),
$$


where $h_{n}$ is the $n^{\text {th }}$ eigenfunction of the harmonic oscillator, defined by

$$
h_{n}(y)=\beta_{n}\left(\partial_{y}-y\right)^{n} e^{-y^{2} / 2}
$$

with the constant $\beta_{n}>0$ chosen such that $\left\|h_{n}\right\|=1$. The operator $R_{+}^{(n)}$ from $L^{2}\left(\mathbb{R}_{x, y}^{2}\right)$ to $L^{2}\left(\mathbb{R}_{x}\right)$ is defined by

$$
u \mapsto\left(R_{+}^{(n)} u\right)(x)=\int h_{n}(y) u(x, y) d y
$$

Note that $R_{+}^{(n)}$ is the adjoint of $R_{-}^{(n)} . R_{-}^{(n)}$ is in $S^{0}\left(\mathbb{R}_{x, \xi}^{2} ; \mathscr{L}\left(\mathbb{C}, B_{v}^{k}\right)\right)$ and $R_{+}^{(n)}$ in $S^{0}\left(\mathbb{R}_{x, \xi}^{2} ; \mathscr{L}\left(B_{y}^{k+2}, \mathbb{C}\right)\right)$. We study the following associated Grushin operator:

$$
\mathscr{P}_{B, V}(x, \check{\zeta})=\left(\begin{array}{cc}
h z-H_{B, V}^{(n)} & R_{-}^{(n)} \\
R_{+}^{(n)} & 0
\end{array}\right),
$$

where

$$
H_{B, V}^{(n)}=D_{y}^{2}+y^{2}-(2 n+1)+h V^{W}\left(x+h^{1 / 2} y, \xi-h^{1 / 2} D_{y}\right)
$$

is in $S^{0}\left(\mathbb{R}_{x, \xi}^{2} ; \mathscr{L}\left(B_{y}^{k+1}, B_{y}^{h}\right)\right)$. The operator $\mathscr{P}$ is in the class $S^{0}\left(\mathbb{R}_{x, \xi}^{2} ; \mathscr{L}\left(B_{y}^{h+1} \times\right.\right.$ $\mathbb{C}, B_{y}^{k} \times \mathbb{C}$ )) for all non-negative integers $k$.

1. The Case $V=0$. We have

$$
\mathscr{P}_{B, 0}^{(n)}(z)=\left(\begin{array}{cc}
(2 n+1)+h z-D_{y}^{2}-y^{2} & R_{-}^{(n)} \\
R_{+}^{(n)} & 0
\end{array}\right) .
$$

For $|h z|<1, \mathscr{P}_{B, 0}^{(n)}(z)$ is invertible [HS], and its inverse, denoted by $\mathscr{E}_{0}^{(n)}(z)$ can be written as

$$
\mathscr{P}_{B, 0}^{(n)}(z)^{-1}=\mathscr{E}_{0}^{(n)}(z)=\left(\begin{array}{cc}
E_{0}^{(n)}(z) & E_{+, 0}^{(n)} \\
E_{-, 0}^{(n)} & E_{-+, 0}^{(n)}(z)
\end{array}\right) .
$$

We decompose $L^{2}\left(\mathbb{R}_{x, y}^{2}\right)$ as follows:

$$
L^{2}\left(\mathbb{R}_{x, y}^{2}\right)=\sum_{l} \mathbb{C} h_{l}(y) \otimes L^{2}\left(\mathbb{R}_{x}\right)=\bigoplus_{l} E_{l} .
$$

It is well known [HS] that $E_{0}^{(n)}(z)$ is a p.d.o. whose Weyl symbol $e_{0}(x, y, \xi, \eta ; z)$ satisfies

$$
e_{0}(x, y, \xi, \eta ; z)=e_{0}(y, \eta ; z)
$$

$e_{0}$ is holomorphic in $z$ in $\mathbf{B}\left(0, h^{-1}\right)$ and is in class $S^{0}$. In the decomposition $\bigoplus_{l} E_{l}$, we have:

$$
\left(E_{0}^{(n)}(z)\right)_{\mathscr{L}\left(E_{l}, E_{k}\right)}=\left(1-\delta_{n, l}\right) \delta_{l, k} /(2(l-n)-z)
$$

(where one has identified $E_{l}$ with $L^{2}\left(\mathbb{R}_{x}\right)$ ). One also has

$$
E_{0,+}^{(n)}=R_{-}^{(n)} ; \quad E_{0,-}^{(n)}=R_{+}^{(n)} ; \quad E_{-+, 0}^{(n)}(z)=-h z .
$$

2. The Case $V \neq 0$. By standard perturbation theory, it can be shown [HS] that there exists an $h_{0}$ such that for all $\left.\left.h \in\right] 0, h_{0}\right], \mathscr{P}_{B, V}^{(n) W}$ is invertible with the inverse $\mathscr{E}^{W}$ 
whose (operator valued) symbol

$$
\mathscr{E}(x, \xi ; z)=\mathscr{P}_{B, i}^{(n)^{-1}}=\left(\begin{array}{cc}
E(x, \xi ; z) & E_{+}(x, \xi ; z) \\
E_{-}(x, \xi ; z) & E_{-+}(x, \xi ; z)
\end{array}\right)
$$

where

$$
\begin{gathered}
h \mathscr{E}(x, \xi) \in S^{0}\left(\mathbb{R}_{x, \xi}^{2} ; \mathscr{L}\left(B_{y}^{k} \times \mathbb{C}, B_{y}^{k+1} \times \mathbb{C}\right)\right), \\
E_{+}(x, \xi) \in S^{0}\left(\mathbb{R}_{x, \xi}^{2} ; \mathscr{L}\left(\mathbb{C}, B_{y}^{k+1}\right)\right), \\
E_{-}(x, \xi) \in S^{0}\left(\mathbb{R}_{x, \xi}^{2} ; \mathscr{L}\left(B_{y}^{k}, \mathbb{C}\right)\right), \\
E_{-+}(x, \xi) / h \in S^{0}\left(\mathbb{R}_{x, \xi}^{2} ; \mathscr{L}(\mathbb{C}, \mathbb{C})\right)=S^{0}\left(\mathbb{R}_{x, \xi}^{2}\right) .
\end{gathered}
$$

$E_{-+}$is called the effective hamiltonian and one has

$$
h z \in \sigma\left(H_{B, V}^{(n)}\right) \Longleftrightarrow 0 \in \sigma\left(E_{-+}\right) .
$$

This is the operator that we shall be studying for the rest of the paper.

If $h z \notin \sigma\left(H_{B, V}^{(n)}\right)$, then

$$
\left(h z-H_{B, V}^{(n)}\right)^{-1}=E(z)-E_{+}(z) E_{-+}^{-1}(z) E_{-}(z) .
$$

Moreover, it can be shown [HS] that there exists $B_{0}=h_{0}^{-1}$ such that for all $B$ with $|B| \geqq B_{0}, E_{-+}$is a symbol analytic in $z$ and $\alpha_{i}\left(i \in \mathbb{Z}^{2}\right)$ for $z \in \mathbf{B}\left(0, h^{-1}\right)$ and $\alpha_{l} \in \mathbf{B}(0, a)$, and is real for $z$ real. $E_{-+}$has an asymptotic expansion in $h$ :

$$
E_{-+}=\sum_{n \geqq 0} h^{n+1} Q_{n}+\mathbf{O}\left(h^{\infty}\right) .
$$

The principal symbol is

$$
Q_{0}(x, \xi ; z)=(V(x, \xi)-z) .
$$

The next term is

$$
Q_{1}(x, \xi ; z)=\frac{2 n+1}{4} \operatorname{Tr} \operatorname{Hess} V(x, \xi),
$$

where Hess denotes the hessian.

\section{The Density of States Measure}

Let $f \in C_{0}(\mathbb{R})$; it can be shown [HS] by using the various Sobolev spaces naturally associated to $P_{B, V}$ that the distribution kernel $K_{f, B}^{(\prime)}$ of $f\left(P_{B, V}^{(\prime)}\right) \in C^{\infty}\left(\mathbb{R}^{2} \times \mathbb{R}^{2}\right)$. Since

$$
\left(\tau_{,}^{B}\right)^{-1} P_{B, V}^{(1)} \tau_{J}^{B}=P_{B, V}^{T_{I^{(1)}}},
$$

we have

$$
\left(\tau_{j}^{B}\right)^{-1} f\left(P_{B, V}^{(\prime)}\right) \tau_{J}^{B}=f\left(P_{B, V}^{T_{,}(1)}\right) .
$$

Let $K_{f, B}^{(\prime)}(x, y)$ be the kernel of $f\left(P_{B, V}^{()}\right)$; we have

$$
K_{f, B}^{(1)}(x+j, x+j)=K_{f, B}^{T,()}(x, x)
$$


for all $j \in \mathbb{Z}^{2}$. By the ergodic theorem, we have

$$
\begin{aligned}
\lim _{L \rightarrow \infty} \frac{1}{(2 L+1)^{2}} \sum_{|j| \leqq L} \int_{x \in \mathbb{E}} K_{f, B}^{T_{,}^{(1)}}(x, x) d x & =\iint_{x \in \mathbb{E}} K_{f, B}^{(1)}(x, x) d x P(d \omega) \quad \text { a.s. } \\
& =\left\langle\int_{E} K_{f, B}(x, x) d x\right\rangle
\end{aligned}
$$

where $L \in \mathbb{N},||$ denotes the sup-norm, $\mathbb{E}$ is the unit square centered at the origin and \langle\rangle is, like before, the expectation with respect to $\omega$. Define

$$
\operatorname{Tr} f\left(P_{B, V}^{(1)}\right)=\lim _{R \rightarrow \infty} \frac{1}{4 R^{2}} \operatorname{Tr}\left(\chi_{R}^{\prime} f\left(P_{B, V}^{(\tilde{)}}\right) \chi_{R}^{\prime}\right)
$$

where $\chi_{R}^{\prime}$ is a $C_{0}^{\infty}$ function on $\mathbb{R}^{2}$ with support in the square of center 0 and length $2 R$ and equal to 1 in the square of center 0 and length $2(R-1)$ with derivatives bounded independently of $R$.

We now show that almost surely $\operatorname{Tr} f\left(P_{B, V}^{\omega}\right)$ exists and is finite. Since for any $R \in(L-1 / 2, L+1 / 2)(L>1)$

$$
\left(\operatorname{Tr} \chi_{R}^{\prime} f\left(P_{B, V}^{(\omega)}\right) \chi_{R}^{\prime}-\operatorname{Tr} \chi_{L+1 / 2}^{\prime} f\left(P_{B, V}^{(1)}\right) \chi_{L+1 / 2}^{\prime}\right) / 4 R^{2}=\mathbf{O}(1 / R),
$$

we only need to show that the limit in the R.H.S. of (3.2) exists along half integers $(R=L+1 / 2)$. Let

$$
D=\int_{|X| \leqq L+1 / 2} K^{(1)}(x, x) d x-\int_{|X| \leqq L+1 / 2} \chi_{R}^{\prime}(x) K^{(1)}(x, x) \chi_{R}^{\prime}(x) d x .
$$

Note that the difference in the integrands has support only for $L-1 / 2 \leqq|x| \leqq$ $L+1 / 2$. We conclude that $|D| \leqq C L$. Therefore the limit in the R.H.S. of $(3.2)$ exists almost surely and we have

$$
\begin{aligned}
\tilde{\operatorname{Tr}} f\left(P_{B, V}^{()}\right) & =(\operatorname{vol} \mathbb{E})^{-1} \int_{\mathbb{E}} K_{f, B}^{(1)}(x, x) P(d \omega) \\
& =(\operatorname{vol} \mathbb{E})^{-1} \int_{\mathbb{E}}\left\langle K_{f, B}(x, x)\right\rangle d x \quad \text { a.s. } \\
& =\left\langle\tilde{\operatorname{Tr}} f\left(P_{B, V}^{()}\right)\right\rangle .
\end{aligned}
$$

We can associate to $\tilde{T} r$ a positive measure $\rho_{B . V}$. It is the density of states measure. We have

$$
\left\langle\tilde{\operatorname{Tr}} f\left(P_{B, V}^{()}\right)\right\rangle=\int f(E) \rho_{B, V}(d E)
$$

for all $f \in C_{0}^{\infty}(\mathbb{R})$.

If $f \in C_{0}^{\infty}(\mathbb{R})$, we can use the usual functional calculus [HS] (see also [S]) to obtain for $A$ self-adjoint

$$
f(A)-\frac{i}{2 \pi} \int \partial_{\bar{z}} \tilde{f}(z)(z-A)^{-1} d \bar{z} \wedge d z,
$$

where $\tilde{f} \in C_{0}^{\infty}(\mathbb{C})$ is an almost analytic extension of $f$, i.e. $\tilde{f}=f$ on $\mathbb{R}$ and $\partial_{\bar{z}} \tilde{f}$ vanishes on $\mathbb{R}$ to infinite order. In order for $f(A)$ to be a pseudo-differential operator, we shall choose $f$ in a restricted class of $h$-dependent functions in $C_{0}^{\infty}$ that will be made more precise later. 
Using the same unitary transformation $U$ as the one we used for $P_{B, V}^{(1)}$, we obtain

$$
\operatorname{Tr} \chi_{R}^{\prime} f\left(P_{B, V}^{(1)}\right) \chi_{R}^{\prime}=\operatorname{Tr} U \chi_{R}^{\prime} U^{-1} U f\left(P_{B, V}^{())}\right) U^{-1} U \chi_{R}^{\prime} U^{-1}
$$

Writing $\chi_{R}=U \chi_{R}^{\prime} U^{-1}$, the symbol of $\chi_{R}$ is $\chi_{R}(x, y, \xi, \eta)=\chi_{R}^{\prime}\left(x+h^{1 / 2} y, \xi-h^{1 / 2} \eta\right)$. We have

$$
\chi_{R}^{W}\left(x+h^{1 / 2} y, \xi-h^{1 / 2} D_{y}\right) \in S^{0}\left(\mathbb{R}_{x, \xi}^{2} ; \mathscr{L}\left(L^{2}\left(\mathbb{R}_{y}\right), B_{y}^{-k}\right)\right)
$$

with $k \geqq 0$. For all $z$ such that $|\operatorname{Im} z| \neq 0$, we have

$$
\left((2 n+1) B+z-\tilde{P}_{B, V}\right)^{-1}=h\left(h z-H_{B, V}\right)^{-1}=h\left[E(z)-E_{+}(z) E_{-+}^{-1}(z) E_{-}(z)\right] .
$$

Since $E(z)$ is holomorphic in $z$, we have

$$
U f\left(P_{B, V}^{(\prime)}\right) U^{-1}=f\left(\tilde{P}_{B, V}^{\omega}\right)=-\frac{i h}{2 \pi} \int \hat{o}_{\bar{z}} \tilde{f} E_{+}(z) E_{-+}^{-1}(z) E_{-}(z) d z \wedge d \bar{z}
$$

as before. Therefore

$$
\operatorname{Tr} \chi_{R}^{\prime} f\left(P_{B, V}^{(1)}\right) \chi_{R}^{\prime}=-\frac{i h}{2 \pi} \operatorname{Tr}\left\{\chi_{R}\left(\int \partial_{\bar{z}} \tilde{f} E_{+}(z) E_{-+}^{-1}(z) E_{-}(z) d \bar{z} \wedge d z\right) \chi_{R}\right\} .
$$

We now show that $\chi_{R} E_{+} E_{-+}^{-1} E_{-} \chi_{R}$ is trace class for $|\operatorname{Im} z| \neq 0$. Hence we will have

$$
\operatorname{Tr} \chi_{R}^{\prime} f\left(P_{B, V}^{(1)}\right) \chi_{R}^{\prime}=-\frac{i h}{2 \pi} \int \partial_{\bar{z}} \tilde{f} \operatorname{Tr}\left(\chi_{R} E_{+} E_{-+}^{-1} E_{-} \chi_{R}\right) d \bar{z} \wedge d z .
$$

Lemma 1. The operator $E_{-} \chi_{R}$ is Hilbert-Schmidt from $L^{2}\left(\mathbb{R}_{x}, L^{2}\left(\mathbb{R}_{y}\right)\right)$ to $L^{2}\left(\mathbb{R}_{x}\right.$, $\mathbb{C})=L^{2}\left(\mathbb{R}_{x}\right)$. Moreover $\left\|E_{-} \chi_{R}\right\|_{H S} \leqq C h^{-1 / 2} R$, where $C$ is a constant independent of $R, h$ and $\omega$.

To prove the above lemma, we need the following well known fact:

Proposition 1. The operator $P^{W}\left(x, h D_{x}\right)$ from $L^{2}\left(\mathbb{R}_{x} ; \mathscr{H}_{1}\right)$ to $L^{2}\left(\mathbb{R}_{x} ; \mathscr{H}_{2}\right)$, where $\mathscr{H}_{1}$ and $\mathscr{H}_{2}$ are arbitrary Hilbert spaces, is Hilbert-Schmidt if and only if

$$
\int\left\|P^{W}(x, \xi)\right\|_{H S\left(\mathscr{H}_{1}, \mathscr{H}_{2}\right)}^{2} d x d \xi<\infty .
$$

Moreover, when $P^{W}\left(x, h D_{x}\right)$ is Hilbert-Schmidt, we have

$$
\left\|P^{W}\left(x, h D_{x}\right)\right\|_{H S}^{2}=\frac{1}{2 \pi h} \int\left\|P^{W}(x, \xi)\right\|_{H S\left(\mathscr{H}_{1}, \mathscr{H}_{2}\right)}^{2} d x d \xi .
$$

Proof of Lemma 1. We consider $E_{-} \# \chi_{R}(x, \xi)$ as the composition:

$$
\mathbb{C} \stackrel{E_{-}}{\longleftarrow} B_{y}^{-k^{\prime}} \stackrel{I}{\longleftarrow} B_{y}^{-k} \stackrel{\chi_{R}}{\longleftarrow} L^{2} \quad k^{\prime}>k>0 .
$$

Let $\Lambda_{-k}$ be the symbol for $\left[1+y^{2}+D_{y}^{2}\right]^{-k}$. It verifies

$$
\left|\partial_{y}^{\alpha} \partial_{\eta}^{\beta} \Lambda_{-k}(y, \eta)\right| \leqq C_{\alpha, \beta}(1+\|(y, \eta)\|)^{-2 k-\alpha-\beta},
$$

for some $C_{x, \beta}$ and where $\|(y, \eta)\|=\left(y^{2}+\eta^{2}\right)^{1 / 2}$. Let

$$
K_{x, \xi}(y, \eta)=\left(\Lambda_{-k} \sharp \chi_{R}\right)(y, \eta) \text {. }
$$


Using the composition formula for two Weyl 1-quantization symbols $a, b$ :

$$
\begin{aligned}
a^{W} \# b^{W}(y, \eta)= & \left(\frac{1}{2 \pi}\right)^{2} \iint e^{l\left(y_{1} \eta_{2}-y_{2} \eta_{1}\right)} a\left(y-y_{1}, \eta-\eta_{1}\right) \\
& \times b\left(y-y_{2}, \eta-\eta_{2}\right) d y_{1} d y_{2} d \eta_{1} d \eta_{2},
\end{aligned}
$$

we have

$$
\begin{aligned}
K_{x, \xi}(y, \eta) & =\frac{1}{4 \pi^{2}} \iint e^{i\left(y_{1} \eta_{2}-y_{2} \eta_{1}\right)} \Lambda_{-k}\left(y-y_{1}, \eta-\eta_{1}\right) \\
& -\chi_{R}\left(x+h^{1 / 2}\left(y-y_{2}\right), \xi-h^{1 / 2}\left(\eta-\eta_{2}\right)\right) d y_{1} d y_{2} d \eta_{1} d \eta_{2} .
\end{aligned}
$$

Since $\chi_{R}$ has support in a square of length $2 R$, we have

$$
\left|\left(y-y_{2}, \eta-\eta_{2}\right)\right| \geqq \frac{1}{C h^{1 / 2}}[|(x, \xi)|-R]
$$

for some $C>0$. Let $|(x, \xi)| \geqq R+1$. Define:

$$
\begin{aligned}
Y & =\left(y_{1}, \eta_{1}, y_{2}, \eta_{2}\right) \\
Y_{1} & =\text { components of } Y \\
Q(Y) & =y_{1} \eta_{2}-\eta_{1} y_{2} \\
L^{t}\left(Y, D_{Y}\right) & =\left(\gamma_{Y}+\left(1-\gamma_{Y}\right)\|\nabla Q(Y)\|^{2}\right)^{-1}\left(\gamma_{Y}+\left(1-\gamma_{Y}\right) \nabla Q(Y) D_{Y}\right),
\end{aligned}
$$

where $\gamma_{Y}$ is a $C_{0}^{\infty}$ function: $\gamma_{Y}=1$ for $\|Y\| \leqq 1-\beta, \gamma_{Y}=0$ for $\|Y\| \geqq 1$, $(0<\beta<1)$ then

$$
L^{t} e^{l Q(Y)}=e^{l Q(Y)} .
$$

By a straightforward computation, we have

$$
L\left(Y, D_{Y}\right)=a_{0}(Y)+\sum_{l=1}^{4} a_{l}(Y) D_{Y_{l}}
$$

where

$$
\begin{aligned}
& \left|\partial^{\alpha} a_{0}\right| \leqq C(\|Y\|+1)^{-2-|\gamma|}, \\
& \left|\partial^{\alpha} a_{J}\right| \leqq C(\|Y\|+1)^{-1-|x|} .
\end{aligned}
$$

Integration by parts $N+5$ times, with $N \geqq k$, we have

$$
K_{x . \zeta}(y, \eta)=\frac{1}{4 \pi^{2}} \iint e^{l Q(Y)} \Gamma_{N,-k, R}^{(x, \xi)}(y, \eta ; Y) d Y,
$$

where

$\Gamma_{N,-h, R}^{(\lambda, \breve{\zeta})}(y, \eta ; Y)=L^{N+5}\left[\Lambda_{-k}\left(y-y_{1}, \eta-\eta_{1}\right) \chi_{R}\left(x+h^{1 / 2}\left(y-y_{2}\right), \xi-h^{1 / 2}\left(\eta-\eta_{2}\right)\right)\right]$.

From (3.5) and the bounds on $\Lambda_{-k}, \chi_{R}$ and their derivatives, we have

$$
\begin{gathered}
\left|\Gamma_{N,-k, R}^{(x, \breve{\zeta})}(y, \eta ; Y)\right| \leqq C_{N}\left(1+y_{1}^{2}+\eta_{1}^{2}+y_{2}^{2}+\eta_{2}^{2}\right)^{-(N+5) / 2}\left(1+\left\|\left(y-y_{1}, \eta-\eta_{1}\right)\right\|\right)^{-2 k} \\
F\left(\left|\left(y-y_{2}, \eta-\eta_{2}\right)\right|-\frac{|(x, \xi)|-R}{C h^{1 / 2}}\right),
\end{gathered}
$$


where $F$ is such that

$$
\begin{array}{ll}
F(u)=1 & \text { if } u \geqq 0, \\
F(u)=0 & \text { otherwise } .
\end{array}
$$

Case 1.

$$
|(y, \eta)| \leqq \frac{|(x, \xi)|-R}{2 C h^{1 / 2}}
$$

From (3.4) we have:

$$
\left|\left(y_{2}, \eta_{2}\right)\right| \geqq \frac{1}{2 C h^{1 / 2}}(|(x, \xi)|-R) .
$$

Therefore

$$
\left|\Gamma_{N}^{(x, \xi)}(y, \eta)\right| \leqq \frac{C_{N}}{\left(1+\|Y\|^{2}\right)^{5 / 2}} \frac{1}{\left[(|(x, \xi)|-R)_{+}+1\right]^{N}}
$$

for all $(x, \xi)$.

Case 2.

$$
|(y, \eta)| \geqq \frac{|(x, \xi)|-R}{2 C h^{1 / 2}},
$$

then

$$
\left|\Gamma_{N}^{(x, \breve{\xi})}(y, \eta ; Y)\right| \leqq \frac{C_{N}}{\left[1+\|Y\|^{2}\right]^{5 / 2}}\left[1+\left|\left(y-y_{1}, \eta-\eta_{1}\right)\right|\right]^{-2 k}\left[1+\left|\left(y_{1}, \eta_{1}\right)\right|\right]^{-N} .
$$

Using (3.7), we have

$$
\left|\Gamma_{N}^{(\lambda, \xi)}(y, \eta ; Y)\right| \leqq \frac{C_{N}}{\left[1+\|Y\|^{2}\right]^{5 / 2}} \frac{1}{\left[(|(x, \xi)|-R)_{+}+1\right]^{\min (N, 2 k)}}
$$

for all $(x, \xi)$.

Hence, taking $N=k$, we have

$$
\left|K_{\imath, \xi}(y, \eta)\right| \leqq \frac{1}{4 \pi^{2}} \int \Gamma_{N}^{(x, \xi)}(y, \eta ; Y) d Y \leqq C_{k}\left[1+(|(x, \xi)|-R)_{+}\right]^{-k}
$$

for all $(x, \zeta)$. Similarly, we have

$$
\left|\partial_{y}^{\alpha} \partial_{\eta}^{\beta} K_{x, \xi}(y, \eta)\right| \leqq C_{k, \alpha, \beta}\left[1+(|(x, \xi)|-R)_{+}\right]^{-k}
$$

for all $\alpha, \beta$ and all $(x, \xi)$. Therefore

$$
\begin{aligned}
& \left\|\chi_{R}^{W}\left(x+h^{1 / 2} y, \xi-h^{1 / 2} D_{y}\right)\right\|_{\mathscr{L}\left(L^{2}\left(\mathbb{R}_{y}\right) \cdot B_{y}^{-h}\right)} \\
& \quad=\left\|\left(1+y^{2}+D_{y}^{2}\right)^{-h} \chi_{R}^{W}\left(x+h^{1 / 2} y, \xi-h^{1 / 2} D_{y}\right)\right\|_{\mathscr{L}\left(L^{2}\left(\mathbb{R}_{y}\right), L^{2}\left(\mathbb{R}_{v}\right)\right)} \quad(k \geqq 0) \\
& \quad \leqq C_{k}\left[1+(|(x, \xi)|-R)_{+}\right]^{-k} .
\end{aligned}
$$

Since the embedding of $B^{m}$ in $B^{n}$ for $m>n+1$ is Hilbert-Schmidt, we have

$$
\begin{aligned}
\left\|\tilde{\chi}_{R}(x, \xi)\right\|_{H S\left(L^{2}, B^{-k^{\prime}}\right)} & \leqq\|I\|_{H S\left(B^{-h}, B^{-k^{\prime}}\right)}\left\|\tilde{\chi}_{R}(x, \xi)\right\|_{\mathscr{L}\left(L^{2}, B^{-k}\right)} \\
& \leqq C_{k, k^{\prime}}\left[1+(|(x, \xi)|-R)_{+}\right]^{-k}
\end{aligned}
$$


for all $k^{\prime}>k+1$ and all $(x, \xi)$, where $\tilde{\chi}_{R}$ is $\chi_{R}$ viewed as an operator valued symbol. Similarly, we have

$$
\left\|\partial_{x}^{\alpha} \partial_{\breve{\zeta}}^{\beta} \tilde{\chi}_{R}(x, \xi)\right\|_{H S\left(L^{2}, B^{-k^{\prime}}\right)} \leqq C_{\alpha, \beta, k, k^{\prime}}\left[1+(|(x, \xi)|-R)_{+}\right]^{-k}
$$

for all $k^{\prime}>k+1$. Since $\chi_{R}$ is chosen such that the bounds on $\chi_{R}$ and its derivatives are independent of $R$, the composition of the symbols:

$$
E_{-} \# \chi_{R} \in S^{0}\left(\mathbb{R}_{x, \zeta \xi}^{2} ; \mathscr{L}\left(L^{2}\left(\mathbb{R}_{y}\right), \mathbb{C}\right)\right)
$$

uniformly in $R$. Hence

$$
\left\|E_{-} \# \tilde{\chi}_{R}(x, \xi)\right\|_{H S\left(L_{y}^{2}, \mathbb{C}\right)} \leqq C_{k}\left[1+(|(x, \xi)|-R)_{+}\right]^{-k}
$$

for all $k>0$. By Proposition 1, we have

$$
\left\|E_{-} \chi_{R}\right\|_{H S} \leqq C h^{-1 / 2} R \text {. }
$$

Proposition 2. The operator $\chi_{R} E_{+} E_{-+}^{-1} E_{-} \chi_{R}$ is trace class on $L^{2}\left(\mathbb{R}_{x}, L^{2}\left(\mathbb{R}_{y}\right)\right)$ for $|\operatorname{Im} z| \neq 0$.

Proof.

$$
\left\|\chi_{R} E_{+} E_{-+}^{-1} E_{-} \chi_{R}\right\|_{\mathrm{T}_{1}} \leqq\left\|\chi_{R} E_{+}\right\|_{H S}\left\|E_{-+}^{-1}\right\|\left\|E_{-} \chi_{R}\right\|_{H S} \leqq\left(C^{2} h^{-1} /|\operatorname{Im} z|\right) R^{2},
$$

where we have used the fact that $\chi_{R} E_{+}$is the adjoint of $E_{-} \chi_{R}$, and is therefore Hilbert-Schmidt.

Using the property of cyclic invariance of the trace, we have

$$
\begin{aligned}
& \operatorname{Tr}\left(\chi_{R} E_{+} E_{-+}^{-1} E_{-} \chi_{R}\right)=\operatorname{Tr}\left(E_{-} \chi_{R}^{2} E_{+} E_{-+}^{-1}\right) \\
& \quad=\operatorname{Tr}\left(\left(\chi_{R}^{(1)}\right)^{2} E_{-} E_{+} E_{-+}^{-1}\right)+\operatorname{Tr}\left(\left[E_{-}, \chi_{R}\right]_{0}\left(\chi_{R} E_{+}\right) E_{-+}^{-1}\right)+\operatorname{Tr}\left(\chi_{R}^{(1)}\left[E_{-}, \chi_{R}\right]_{0} E_{+} E_{-+}^{-1}\right) \\
& \quad=\operatorname{Tr} A_{1}+\operatorname{Tr} A_{2}+\operatorname{Tr} A_{3},
\end{aligned}
$$

where $\chi_{R}^{(1)}(x, \xi)=\chi_{R}(x, \xi)$ and we have written

$$
\left[E_{-}, \chi_{R}\right]_{0}=E_{-} \chi_{R}-\chi_{R}^{(1)} E_{-} .
$$

By the classical equality:

$$
\left\|\chi_{R}^{(1)}\right\|_{H S}^{2}=\frac{1}{2 \pi h} \int\left|\chi_{R}(x, \xi)\right|^{2} d x d \xi
$$

we obviously have:

Lemma 2. $\chi_{R}^{(1)}$ is Hilbert-Schmidt, and

$$
\left\|\chi_{R}^{(1)}\right\|_{H S} \leqq C h^{-1 / 2} R
$$

Corollary. $A_{1}=\left(\chi_{R}^{(1)}\right)^{2} E_{-} E_{+} E_{-+}^{-1}$ is trace-class for $|\operatorname{Im} z| \neq 0$ :

$$
\operatorname{Tr}\left(\left(\chi_{R}^{(1)}\right)^{2} E_{-} E_{+} E_{-+}^{-1}\right)=\operatorname{Tr}\left(\chi_{R}^{(1)} E_{-} E_{+} E_{-+}^{-1} \chi_{R}^{(1)}\right) .
$$

Furthermore, $\operatorname{Tr} A_{1} \leqq C h^{-1}|\operatorname{Im} z|^{-1} R^{2}$, with $C$ independent of $R, h$ and $\omega$. 
Lemma 3. $A_{2}$ and $A_{3}$ are trace-class and

$$
\operatorname{Tr} A_{2} \leqq C h^{-1}|\operatorname{Im} z|^{-1} R^{3 / 2} \quad \operatorname{Tr} A_{3} \leqq C h^{-1}|\operatorname{Im} z|^{-1} R^{3 / 2},
$$

where $C$ is independent of $R, h$ and $\omega$.

Proof. We already know that

$$
\left\|\left[E_{-}, \chi_{R}\right]_{0}\right\|_{H S} \leqq C h^{-1 / 2} R .
$$

We now show that, in fact

$$
\left\|\left[E_{-}, \chi_{R}\right]_{0}\right\|_{H S} \leqq C h^{-1 / 2} R^{1 / 2} .
$$

Let $\chi_{R}^{c}=1-\chi_{R}$. Replacing $\tilde{\chi}_{R}$ by $\tilde{\chi}_{R}^{c}$ in (3.8), we have

$$
\mid E_{-} \# \tilde{\chi}_{R}^{c}(x, \xi) \|_{H S\left(L_{y}^{2}, \mathbb{C}\right)} \leqq C_{h}\left[1+(R-|(x, \xi)|)_{+}\right]^{-k} \text { for all } k>0 \text {. }
$$

Let $\chi_{R}^{(1) c}=1-\chi_{R}^{(1)}$. Viewing $\chi_{R}^{(1) c} \# E_{-}(x, \xi)$ as the composition of operators

$$
\mathbb{C} \stackrel{\chi_{R}^{(1) c}}{\longleftarrow} \mathbb{C} \stackrel{E_{-}}{\longleftarrow} B^{-k^{\prime}} \stackrel{I}{\longleftarrow} L^{2} \quad k^{\prime}>0,
$$

and using the fact that the embedding of $L^{2}$ in $B^{-k^{\prime}}$ is Hilbert-Schmidt for $k^{\prime}>1$, we have

$$
\begin{aligned}
\left\|\chi_{R}^{(1) c} \# E_{-}(x, \xi)\right\|_{H S\left(L_{y}^{2}, \mathbb{C}\right)} & \leqq C_{k^{\prime}}\left\|\chi_{R}^{(1) c} \# E_{-}(x, \xi)\right\|_{\mathscr{L}\left(B^{-k^{\prime}}, \mathbb{C}\right)}\|I\|_{H S\left(L^{2}, B^{-h^{\prime}}\right)} \\
& \left.\leqq C_{k}[1+R-|(x, \xi)|)_{+}\right]^{-k}
\end{aligned}
$$

for all $k>0$, where we used the composition formula for two symbols. Hence from (3.11) and (3.12),

$$
\left\|\left[E_{-}, \chi_{R}^{c}\right]_{0}(x, \xi)\right\|_{H S} \leqq C_{k}\left[1+(R-|(x, \xi)|)_{+}\right]^{-k} \quad k>0 .
$$

Since

and

$$
\left[E_{-}, \chi_{R}\right]_{0}=-\left[E_{-}, \chi_{R}^{c}\right]_{0}
$$

$$
\left\|\left[E_{-}, \chi_{R}\right]_{0}(x, \xi)\right\|_{H S} \leqq C_{k}\left[1+(|(x, \xi)|-R)_{+}\right]^{-h} \quad k>0,
$$

we have

$$
\left\|\left[E_{-}, \chi_{R}\right]_{0}(x, \xi)\right\|_{H S\left(L_{y}^{2}, \mathbb{C}\right)} \leqq C_{k}[1+\|(x, \xi)|-R|]^{-k} \quad k>0 .
$$

Therefore

$$
\left\|\left[E_{-}, \chi_{R}\right]_{0}\right\|_{H S\left(L^{2}\left(\mathbb{R}_{1} ; L_{y}^{2}\right), L^{2}\left(\mathbb{R}_{1}\right)\right)} \leqq C h^{-1 / 2} R^{1 / 2} .
$$

This yields the results for $\operatorname{Tr} A_{2}$ and $\operatorname{Tr} A_{3}$.

We now put $\operatorname{Tr} f\left(P_{B, V}^{(1)}\right)$ in a convenient form, which we will use later to compute the asymptotics.

\section{Proposition 3.}

$$
\begin{aligned}
\tilde{\operatorname{Tr}} f\left(P_{B, V}^{()}\right) & =\left\langle\tilde{\operatorname{Tr}} f\left(P_{B, V}^{()}\right)\right\rangle \\
& =-\frac{1}{4 \pi^{2} i h} \int_{\mathbb{E}} \hat{\partial}_{\bar{z}} \tilde{f}\left\langle\left[\hat{\sigma}_{\bar{z}} E_{-+} \#_{h} E_{-+}^{-1}\right](x, \xi, z)\right\rangle(d \bar{z} \wedge d z) d x d \xi \quad \text { a.s. },
\end{aligned}
$$

where $\mathbb{E}$ is a unit square. 
Since $E_{-+}$is holomorphic in $z$, it is now enough to control $E_{-+}^{-1}$ when $|\operatorname{Im} z| \rightarrow 0$.

Proof of Proposition 3. Combining (3.2), (3.3), (3.10), the corollary to Lemma 2 and Lemma 3, we have

$$
\begin{aligned}
\tilde{\operatorname{Tr}} f\left(P_{B, V}^{(1)}\right)= & \left\langle\tilde{\operatorname{Tr}} f\left(P_{B, V}^{(1)}\right)\right\rangle \\
= & \lim _{R \rightarrow \infty} \frac{-1}{16 i \pi^{2} R^{2} h} \int \partial_{\bar{z}} \tilde{f}\left\langle\left[\chi_{R}^{(1)} \#_{h} \partial_{z} E_{-+} \#_{h} E_{-+}^{-1} \#_{h} \chi_{R}^{(1)}\right](x, \xi, z)\right\rangle \\
& \times(d \bar{z} \wedge d z) d x d \xi \quad \text { a.s., }
\end{aligned}
$$

where we used the identity (see [HS])

$$
h^{-1} \partial_{z} E_{-+}=E_{-} \sharp E_{+} .
$$

Let $T=\partial_{z} E_{-+} \#_{h} E_{-+}^{-1}, \mathscr{T}=\chi_{R}^{(1)} \#_{h} T \#_{h} \chi_{R}^{(1)}$. For convenience, we view $\mathscr{T}$ as $\mathscr{T} I$, where $I$ is the identity operator from $L^{2}(\mathbb{R})$ to $L^{2}(\mathbb{R})$, with symbol the function 1. Using the composition formula for four Weyl h-quantization symbols, we have (writing $X=(x, \xi)$ )

$$
\mathscr{T}(X)=\frac{1}{(2 \pi h)^{4}} \int e^{l Q_{4}(Y) / h} \chi_{R}^{(1)}\left(X-Y_{1}\right) T\left(X-Y_{2}\right) \chi_{R}^{(1)}\left(X-Y_{3}\right) \mathbf{1}\left(X-Y_{4}\right) d Y,
$$

where $Y=\left(Y_{1}, Y_{2}, Y_{3}, Y_{4}\right), Y_{l}=\left(y_{l}, \eta_{l}\right), d Y=\prod_{l} d y_{i} d \eta_{l}$ and

$$
Q_{4}(Y)=\sigma\left(Y_{1}, Y_{2}\right)+\sigma\left(Y_{2}, Y_{3}\right)+\sigma\left(Y_{3}, Y_{4}\right)+\sigma\left(Y_{1}, Y_{4}\right)+\sigma\left(Y_{4}, Y_{2}\right)+\sigma\left(Y_{3}, Y_{1}\right) ;
$$

$\sigma$ is the canonical symplectic form:

$$
\sigma\left(Y_{l}, Y_{J}\right)=y_{J} \eta_{l}-y_{l} \eta_{J} .
$$

We first treat the case $|X|>R+1$. Noting that $Q_{4}(Y) / h=Q_{4}\left(h^{-1 / 2} Y\right)$, we see that

$$
\frac{L^{t}}{i}\left(h^{-1 / 2} Y, h^{1 / 2} D_{Y}\right) e^{l Q_{4} / h}=e^{i Q_{4} / h},
$$

where $L^{t}$ is as defined in (3.5) with obvious modifications. By straightforward computation, we have that

$$
\left\|\nabla Q_{4}(Y)\right\|^{2}=\left\|Y^{\prime}\right\|^{2}
$$

where

$$
\begin{gathered}
Y^{\prime}=\left(\begin{array}{cc}
A & O \\
0 & -A
\end{array}\right) Y, \\
Y=\left(\begin{array}{l}
y_{1} \\
y_{2} \\
y_{3} \\
y_{4} \\
\eta_{1} \\
\eta_{2} \\
\eta_{3} \\
\eta_{4}
\end{array}\right),
\end{gathered}
$$


and similarly for $Y^{\prime}$, and

$$
A=\left(\begin{array}{cccc}
0 & -1 & 1 & -1 \\
1 & 0 & -1 & 1 \\
-1 & 1 & 0 & -1 \\
1 & -1 & 1 & 0
\end{array}\right)
$$

$A$ is clearly invertible, hence $\|Y\|^{2} / C \leqq\left\|Y^{\prime}\right\|^{2} \leqq C\|Y\|^{2}$ for some constant $C>0$. Therefore

$$
L=b_{0}\left(h^{-1 / 2} Y\right)+\sum_{l=1}^{8} b_{l}\left(h^{-1 / 2} Y\right) h^{1 / 2} D_{Y_{l}}
$$

with $b_{0}, b_{i}(i \neq 0)$ satisfy the same estimate as $a_{0}, a_{i}(i \neq 0)$ in (3.6). Using the estimates on $b_{0}, b_{i}(i \neq 0)$, we obtain that

$$
L^{N}=\sum_{|\beta| \leqq N} b_{\beta}^{N}\left(h^{-1 / 2} Y\right)\left(h^{1 / 2} D_{Y}\right)^{\beta}
$$

with

$$
\left|\partial^{\alpha} b_{\beta}^{N}(Y)\right| \leqq C(\|Y\|+1)^{-2 N+|\beta|-|x|} .
$$

We therefore have, for all $N \in \mathbb{N}$,

$$
\mathscr{T}(X)=\frac{1}{(2 \pi h)^{4}} \int e^{l Q_{4}(Y) / h} L^{N}\left[\chi_{R}^{(1)}\left(X-Y_{1}\right) T\left(X-Y_{2}\right) \chi_{R}^{(1)}\left(X-Y_{3}\right) I\left(X-Y_{4}\right)\right] d Y .
$$

Since $|X|>R+1$, we have $\left|Y_{1}\right|>(|X|-R) / C,\left|Y_{3}\right|>(|X|-R) / C$. Since

$$
\|T\| \leqq \frac{C}{|\operatorname{Im} z|}
$$

with $C$ independent of $\omega$, we have by Beals' Lemma [Bea] as stated in [S],

$$
\left|\partial_{x}^{\alpha} \partial_{=}^{\beta} T\right| \leqq C_{\alpha, \beta} \max \left(1,\left(\frac{h}{|\operatorname{Im} z|^{2}}\right)^{3}\right)|\operatorname{Im} z|^{-1-\alpha-\beta},
$$

where $C_{x, \beta}$ is independent of $\omega$. Hence using (3.13), (3.14) and taking $N>8$, we have

$$
|\mathscr{T}(X)| \leqq \frac{C_{N}}{(|X|-R)^{N-8}|\operatorname{Im} z|^{7+N}} .
$$

(We do not keep track of powers of $h$, since it is not important here.) For $|X|<$ $R-1$, we write

$$
\begin{aligned}
\mathscr{T}(X)= & \left.\frac{1}{(2 \pi h)^{4}} \int e^{l Q_{4}(Y) / h} \mathbf{1}\left(X-Y_{1}\right) T\left(X-Y_{2}\right) \mathbf{1}\left(X-Y_{3}\right) \mathbf{1}\left(X-Y_{4}\right)\right] d Y \\
& \left.-\frac{1}{(2 \pi h)^{4}} \int e^{l Q_{4}(Y) / h}\left(\mathbf{1}-\chi_{R}^{(1)}\right)\left(X-Y_{1}\right) T\left(X-Y_{2}\right) \mathbf{1}\left(X-Y_{3}\right) \mathbf{1}\left(X-Y_{4}\right)\right] d Y \\
& \left.-\frac{1}{(2 \pi h)^{4}} \int e^{l Q_{4}(Y) / h} \mathbf{1}\left(X-Y_{1}\right) T\left(X-Y_{2}\right)\left(\mathbf{1}-\chi_{R}^{(1)}\right)\left(X-Y_{3}\right) \mathbf{1}\left(X-Y_{4}\right)\right] d Y \\
& +\frac{1}{(2 \pi h)^{4}} \int e^{l Q_{4}(Y) / h}\left(\mathbf{1}-\chi_{R}^{(1)}\right)\left(X-Y_{1}\right) T\left(X-Y_{2}\right)\left(\mathbf{1}-\chi_{R}^{(1)}\right)\left(X-Y_{3}\right) \\
& \left.\times \mathbf{1}\left(X-Y_{4}\right)\right] d Y .
\end{aligned}
$$


The last three terms in the sum are bounded by

$$
\frac{C_{N}}{(R-|X|)^{N-8}|\operatorname{Im} z|^{7+N}}
$$

with $C_{N}$ independent of $\omega$, by using the same method as before. The first term is precisely

$$
T(X)=\partial_{z} E_{-+} \#_{h} E_{-+}^{-1}(X) .
$$

For $R-1<|X|<R+1, \mathscr{T}(X)$ is bounded for $|\operatorname{Im} z| \neq 0$. Hence upon taking the limit $R \rightarrow \infty$ and taking into account the periodicity of $\left\langle\partial_{z} E_{-+} \#_{h} E_{-+}^{-1}(x, \xi)\right\rangle$, we obtain the proposition.

\section{Study of the Operator $f\left(P_{B, V}^{\omega}\right)$}

Let $S_{\partial}^{k}$ denote the class of symbols $a$ such that

$$
\left|\partial_{x}^{\alpha} \partial_{\xi}^{\beta} a(x, \xi ; h)\right| \leqq C_{\gamma, \beta} h^{-k-\delta(x+\beta)} .
$$

For $|\operatorname{Im} z| \geqq h^{\delta}(0<\delta<1 / 2), h E_{-+}^{-1}$ is a p.d.o. whose symbol admits the asymptotic expansion:

$$
h E_{-+}^{-1} \sim \sum_{j \geqq 0} F_{j}
$$

where

$$
\begin{aligned}
& F_{J}=\frac{h^{j} \tilde{Q}_{J}}{(V-z)^{2 j}} \in S_{\delta}^{-\lambda(1-2 \delta)}=S_{\delta}^{k J} \quad(j>0), \\
& F_{0}=\frac{1}{V-z} \in S_{\delta}^{\delta}=S_{\delta}^{k_{0}}, \\
& h E_{-+}^{-1}-\sum_{j=0}^{N} F_{j} \in S_{\delta}^{k_{N+1}} \quad \text { for all } N \in \mathbb{N} .
\end{aligned}
$$

$\tilde{Q}_{\text {J }}$ are functions of the $Q_{i}(i \leqq j)$ and their derivatives; in particular, $\tilde{Q}_{1}=-Q_{1}$. (Recall that $E_{-+}=\sum_{n \geqq 0} h^{n+1} Q_{n}+\mathbf{O}\left(h^{\infty}\right)$.) Since $\partial_{z} E_{-+}$is also in class $S^{0}$ and has the asymptotic expansion:

$$
\hat{O}_{z} E_{-+}^{(1)}=h\left(1+h S_{1}^{(1)}+h^{2} S_{2}^{(1)}+\cdots\right)
$$

with $S_{i} \in S^{0}$ for all $\omega, \partial_{z} E_{-+}^{(1)} \#_{h}\left(E_{-+}^{(1)}\right)^{-1}$ admits the following asymptotic expansion:

$$
\partial_{z} E_{-+}^{()} \#_{h} E_{-+}^{(1)}{ }^{-1}=\frac{1}{V^{(1)}-z}+h\left(\frac{S_{1}^{(1)}}{V^{(1)}-z}+\frac{\tilde{Q}_{1}^{(1)}}{\left(V^{(1)}-z\right)^{2}}\right)+\cdots
$$

for $|\operatorname{Im} z| \geqq h^{\delta}(0<\delta<1 / 2)$.

Using the random character of the parameters $\alpha_{l}$, we have:

Proposition 4. For all $N \in \mathbb{N}, C>0$ there exists $h_{0}$, such that for all $\left.\left.h \in\right] 0, h_{0}\right]$, all $z$ satisfying $|z|<h^{-1}$ with $|\operatorname{Re} z| \geqq 1 / C$ and $|\operatorname{Im} z| \geqq h^{N} / C,\left\langle\partial_{z} E_{-+} \#_{h} E_{-+}^{-1}\right\rangle$ is 
in class $S^{0}$, i.e. $\left|\partial_{x}^{\alpha} \partial_{y}^{\beta}\left\langle\left(\partial_{z} E_{-+} \sharp_{h} E_{-+}^{-1}\right)(x, \xi, z)\right\rangle\right| \leqq C_{N, x, \beta}$. Moreover, $\left\langle\partial_{z} E_{-+} \#_{h} E_{-+}^{-1}\right\rangle$ is a classical symbol in $S_{0}$, i.e. there exist $A_{j} \in S_{0}$, such that

$$
\left\langle\partial_{z} E_{-+} \#_{h} E_{-+}^{-1}\right\rangle=\sum_{j \geqq 0} h^{j} A_{J}+\mathbf{O}\left(h^{\infty}\right),
$$

where $A, \in S^{0}$.

We shall prove the above proposition by scaling: we gradually take into account the potentials at larger and larger length scales. Since $\left\langle\partial_{z} E_{-+} \#_{h} E_{-+}^{-1}\right\rangle$ is $\mathbb{Z}^{2}$ translationally invariant, we only need to prove Proposition 4 for $(x, \xi) \in \mathbb{E}$, where $\mathbb{E}$ is the unit square centered at $(0,0)$.

Without loss of generality, we may assume $v$ is a $C_{0}^{\infty}$ function with support contained in the square centered at $(0,0)$ and of length $b$, with $b \in \mathbb{N}$. Let $\Lambda_{n}$ be an increasing sequence of squares centered at $(0,0)$ of length $l_{n}=2^{n} b+2$. Let $\Lambda_{n}^{\prime}, \Lambda_{n}^{\prime \prime}$ be two corresponding sequences of squares centered at $(0,0)$ of lengths $l_{n}^{\prime}=l_{n}-b$ and $l_{n}^{\prime \prime}=l_{n}+b$ respectively. Clearly for all $(x, \breve{\zeta}) \in \mathbb{E}$, $\operatorname{dist}\left((x, \check{\zeta}), \partial \Lambda_{n}^{\prime}\right) \geqq\left(l_{n}^{\prime}-\right.$ 1) $/ 2$, where $\partial \Lambda_{n}^{\prime}$ is the boundary of $\Lambda_{n}^{\prime}$. Let $H_{n}$ be the reduced operator when $V_{n}(\bar{x})=\sum_{l \in \Lambda_{n}} \alpha_{l} v(\bar{x}-i)\left(E_{-+}\right.$is the reduced operator when $V(\bar{x})=\sum_{i \in \mathbb{Z}^{2}} \alpha_{l} v(\bar{x}-$ $i)$ ). The principal symbol for $H_{n}$ is clearly $h\left(V_{n}(x, \xi)-z\right)$. We write

$$
\begin{aligned}
E_{-+}^{-1} & =H_{0}^{-1}+H_{1}^{-1}-H_{0}^{-1}+H_{2}^{-1}-H_{1}^{-1}+\cdots+H_{N_{0}}^{-1}-H_{N_{0}-1}^{-1}+E_{-+}^{-1}-H_{N_{0}}^{-1} \\
& =H_{0}^{-1}+H_{0}^{-1} \Delta_{1} H_{1}^{-1}+H_{1}^{-1} \Delta_{2} H_{2}^{-1}+\cdots+H_{N_{0}}^{-1} \Delta H_{N_{0}-1}^{-1}+H_{N_{0}}^{-1} \tilde{\Delta}_{N_{0}+1} E_{-+}^{-1},
\end{aligned}
$$

where $\Delta_{n}=H_{n-1}-H_{n}, \tilde{\Delta}_{N_{0}+1}=H_{N_{0}}-E_{-+}$and we used the resolvent equation: $A^{-1}-B^{-1}=B^{-1}(B-A) A^{-1} ; N_{0}$ is yet to be determined. Hence:

$$
\begin{aligned}
\partial_{z} E_{-+} E_{-+}^{-1}= & \left(\partial_{z} E_{-+}\right) H_{0}^{-1}+\left(\partial_{z} E_{-+}\right) H_{0}^{-1} \Delta_{1} H_{1}^{-1}+\left(\partial_{z} E_{-+}\right) H_{1}^{-1} \Delta_{2} H_{2}^{-1} \\
& +\cdots+\left(\partial_{z} E_{-+}\right) H_{N_{0}}^{-1} \tilde{\Delta}_{N_{0}+1} E_{-+}^{-1} .
\end{aligned}
$$

To prove the above proposition, we need to complex dilate in $\alpha$. The Grushin problem remains well posed and we have the following:

Lemma 4. Let $\left.h \in] 0, h_{0}\right]$. Suppose $z$ is such that $|z|<h^{-1}$, and $\alpha$ is such that $|\alpha|_{\infty} \leqq 2 R_{0}$ for some $R_{0}>0$, then for $t$ such that $|t|_{\infty} \leqq R_{0}$, we have

$$
\begin{aligned}
\left|\left\langle\nabla_{\alpha} H_{n}(\alpha), t\right\rangle\right| & \leqq \sup _{|w|=1}\left|H_{n}(\alpha+w t)\right|, \\
\left|\left\langle\nabla_{\alpha} E_{-+}(\alpha), t\right\rangle\right| & \leqq \sup _{|w|=1}\left|E_{-+}(\alpha+w t)\right| .
\end{aligned}
$$

Proof. We prove the second assertion, the first can be proved in the same way. We have

$$
\left\langle\nabla_{\alpha} E_{-+}(\alpha), t\right\rangle=\left.\frac{\partial}{\partial w} E_{-+}(\alpha+w t)\right|_{w=0}
$$

Hence

$$
\left|\left\langle\nabla_{\alpha} E_{-+}(\alpha), t\right\rangle\right| \leqq \sup _{|w|=1}\left|E_{-+}(\alpha+w t)\right|
$$

by the Cauchy inequality. 
For $z$ such that $\operatorname{Re} z \operatorname{Im} z<0(\operatorname{Re} z \operatorname{Im} z>0)$, we define $V_{\theta}=e^{i \theta} V\left(V_{\theta}=e^{-l \theta} V\right)$ $(\theta>0)$. Let $E_{-+}(\theta), H_{n}(\theta)$ be the corresponding reduced operator.

Corollary. $h^{-1} \partial E_{-+}(\theta) / \partial \theta$ and $h^{-1} \partial H_{n}(\theta) / \partial \theta$ are bounded symbols in $S_{0}$.

From [HS] we have the following asymptotic expansion for $H_{n}$ :

$$
H_{n}(x, \xi, h, z)=\sum_{J \geqq 0} \bar{Q}_{J}^{(n)}(x, \xi, g, z, h) h^{j+1}-h z,
$$

with

$$
\begin{aligned}
g & =h^{1 / 2}, \\
\bar{Q}_{J}^{(n)}(x, \xi, g, z) & =(-1)^{J} \int h_{m}(y)\left(u_{J}^{(n)}\left(y, x, D_{y}, \xi, g\right) h_{m}\right)(y) d y, \\
u_{j}^{(n)}\left(y, x, D_{y}, \xi\right) & =V_{n}^{W}\left[x+g y, \xi-g D_{y}\right] \#_{h}\left(e_{0}\left(y, D_{y}, z\right) V_{n}^{W}\right)^{\prime},
\end{aligned}
$$

and $e_{0}\left(y, D_{y}, z\right)$ is the symbol of the operator $E_{0}^{(n)}$ as in Sect. II.1. $E_{-+}$corresponds to taking the potential to be $V$. By symmetry arguments, it is easy to see that

$$
\bar{Q}_{j}^{(n)}(x, \xi, g, z)=\bar{Q}_{j}^{(n)}(x, \xi,-g, z) .
$$

So $H_{n}(x, \xi, g, z)$ has an asymptotic expansion in $h\left(=g^{2}\right)$,

$$
H_{n}(x, \xi, h, z)=\sum_{j \geqq 0} Q_{j}^{(n)}(x, \xi, z, h) h^{j+1}-h z
$$

It is easy to see that $Q_{j}^{(n)}(x, \xi)$ only depends on the potential and its derivatives at $(x, \xi)$. We deduce that:

$$
\left|\partial_{x}^{\chi} \partial_{\xi}^{\beta} \Delta_{n}(x, \xi)\right| \leqq C_{\alpha, \beta}^{(N)} h^{N}
$$

for all $N,(x, \xi)$ in $\Lambda_{n-1}^{\prime}$ or $\mathbb{R}^{2} \backslash \Lambda_{n}^{\prime \prime}$ and $C_{\alpha, \beta}^{(N)}$ is independent of $\omega$. (Recall that $\Delta_{n}=H_{n-1}-H_{n}$.)

Proof of Proposition 4. We use the expansion (4.1). We assume $\operatorname{Re} z \operatorname{Im} z<0$, the other case can clearly be treated in a parallel manner. We first estimate the term $\left\langle\partial_{z} E_{-+} H_{n-1}^{-1} \Delta_{n} H_{n}^{-1}\right\rangle$. We complex dilate: $\alpha_{l} \rightarrow \alpha_{l} e^{i \theta_{n}}$ with $\theta_{n}=1 / l_{n}$ for all $i \in \Lambda_{n}$. We see that for $n$ such that $1 / l_{n} \leqq|\operatorname{Im} z|$, complex dilation will not give us a better estimate on $H_{n}^{-1}$. Hence for a given $N, N_{0}$ is chosen to be the largest integer such that $1 / l_{N_{0}} \geqq|\operatorname{Im} z| \geqq h^{N} / C ; \quad N_{0}$ is therefore at most of order $N \log (1 / h)$. Let

$$
\left\langle\partial_{z} E_{-+} \#_{h} H_{n-1}^{-1} \#_{h} \Delta_{n} \#_{h} H_{n}^{-1}\right\rangle_{c} \stackrel{\text { def }}{=} \int_{\Gamma(0)} \partial_{z} E_{-+} \#_{h} H_{n-1}^{-1} \#_{h} \Delta_{n} \#_{h} H_{n}^{-1} \mathscr{G}(\alpha) d \alpha,
$$

where $\alpha=\left\{\alpha_{i}\right\}_{i \in \Lambda_{n}}, d \alpha=\prod_{l \in \Lambda_{n}} d \alpha_{i}, \mathscr{G}(\alpha)=\prod_{l \in \Lambda_{n}} g\left(\alpha_{l}\right)$, i.e. the LHS is the conditional expectation value conditioned upon $\alpha_{l} \notin \Lambda_{n}$. Then

$$
\left|\left\langle\partial_{z} E_{-+} \#_{h} H_{n-1}^{-1} \#_{h} \Delta_{n} \#_{h} H_{n}^{-1}\right\rangle\right| \leqq\left|\left\langle\partial_{z} E_{-+} \#_{h} H_{n-1}^{-1} \#_{h} \Delta_{n} \#_{h} H_{n}^{-1}\right\rangle_{c}\right|_{\infty},
$$


where $\left.\right|_{\infty}$ is the $L^{\infty}$-norm with respect to $\alpha_{l}, i \notin \Lambda_{n}$. Hence it is enough to estimate $\left|\left\langle\partial_{z} E_{-+} \#_{h} H_{n-1}^{-1} \#_{h} \Delta_{n} \#_{h} H_{n}^{-1}\right\rangle_{c}\right|$. By the Stokes formula, we have

$$
\begin{aligned}
\left\langle\partial_{z} E_{-+} \#_{h} H_{n-1}^{-1} \sharp_{h} \Delta_{n} \#_{h} H_{n}^{-1}\right\rangle_{c}= & \int_{\Gamma(0)} \partial_{z} E_{-+} \#_{h} H_{n-1}^{-1} \#_{h} \Delta_{n} \#_{h} H_{n}^{-1} \mathscr{G}(\alpha) d x \\
= & \int_{\Gamma\left(0_{n}\right)} \partial_{z} E_{-+} \#_{h} H_{n-1}^{-1} \#_{h} \Delta_{n} \#_{h} H_{n}^{-1} \tilde{\mathscr{G}}(a) d a \\
& +\int_{\Omega\left(0_{n}\right)} \sum_{j=1}^{\left|A_{n}\right|} \frac{\partial \tilde{C}}{\partial \bar{a}_{j}}\left[\partial_{z} E_{-+} \#_{h} H_{n-1}^{-1} \#_{h} \Delta_{n} \#_{h} H_{n}^{-1}\right] d \bar{a}_{J} \wedge d a,
\end{aligned}
$$

where $a=\left\{\alpha_{l} e^{l()}\right\}_{l \in \Lambda_{n}}=\left\{a_{l}\right\}_{l \in \Lambda_{n}}, \tilde{\mathscr{G}}$ is an almost analytic extension of $\mathscr{G}$, such that $\partial \tilde{\mathscr{G}} /\left.\partial \bar{a}\right|_{\mathbb{R}^{\Lambda n}}=0$ to infinite order and $\Omega\left(\theta_{n}\right)$ is the volume enclosed by $\Gamma(0), \Gamma\left(\theta_{n}\right)$ and $\left|\alpha_{l}\right|=p$ for all $i \in \Lambda_{n}$. (Recall that supp $g \subset(-p, p)$.) We now choose

$$
\tilde{\mathscr{G}}(a)=\prod_{i \in \Lambda_{n}} \tilde{g}\left(a_{i}\right)
$$

where $\tilde{g}\left(a_{l}\right)$ is an almost analytic extension of $g\left(\alpha_{l}\right)$ such that $\tilde{g}\left(a_{l}\right) \in C_{0}^{\infty}(\mathbb{C})$ and $\partial \tilde{g} /\left.\partial \bar{a}_{l}\right|_{\mathbb{R}}=0$ to infinite order.

Let $h S_{0, z}=h^{-1} H_{n}(\theta)-e^{l \theta} V_{n}+z,\left(0 \leqq \theta \leqq \theta_{n}\right)$, (Note that $S_{(0, z} \in S_{0}$.), then

$$
h^{-1}\left\langle\phi, H_{n}(\theta) \phi\right\rangle=e^{l()} A_{0}+h\left\langle\phi, S_{(,, z} \phi\right\rangle-z,
$$

where

$$
A_{0}=\frac{1}{2 \pi h} \int \bar{\phi}(x) e^{\frac{(2-y) \xi}{h}} \phi(y) V_{n}\left(\frac{x+y}{2}, \xi\right) d x d y d \xi
$$

is real. Since $\partial_{0} S$ and $\partial_{z} S$ are in $S^{0},\left\langle\phi, S_{0, z} \phi\right\rangle$ is real when $\theta=0, \operatorname{Im} z=0$ we have

$$
\operatorname{Im}\left|\left\langle\phi, S_{0, z} \phi\right\rangle\right| \leqq C(\theta+|\operatorname{Im} z|)\|\phi\|,
$$

where $C$ is independent of $\omega$. Hence there exists $h$ sufficiently small, such that for $z$ with $|\operatorname{Re} z|>c^{\prime}$,

$$
\operatorname{dist}\left(0, \mathscr{N} \mathscr{R}_{H_{n}(\theta)}\right) \geqq \operatorname{ch}(\theta+|\operatorname{Im} z|),
$$

where $\mathscr{N} \mathscr{R}_{H_{n}(\theta)}$ denotes the numerical range of $H_{n}(\theta)$ and $c$ is independent of $\omega$. We therefore have

$$
\left\|H_{n}^{-1}(\theta)\right\| \leqq \frac{1}{\operatorname{ch}(\theta+|\operatorname{Im} z|)}
$$

Similarly,

$$
\left\|H_{n-1}^{-1}(\theta)\right\| \leqq \frac{1}{\operatorname{ch}(\theta+|\operatorname{Im} z|)} .
$$

Using Beals' lemma, we have

$$
h\left|\partial_{1}^{\alpha} \partial_{\xi}^{\beta}\left(\begin{array}{c}
H_{n-1}^{-1} \\
H_{n}^{-1}
\end{array}\right)\right| \leqq C_{\alpha, \beta} \max \left(1,\left(\frac{h}{(\theta+|\operatorname{Im} z|)^{2}}\right)^{3}\right)(\theta+|\operatorname{Im} z|)^{-1-\alpha-\beta},
$$

where $C_{\gamma, \beta}$ is independent of $\omega$. We now show that $\left(\partial_{z} E_{-+}\right) \sharp_{h} H_{n-1}^{-1} \sharp_{h} \Delta_{n} \sharp_{h} H_{n}^{-1}(x, \xi)$ is small for $(x, \xi) \in \mathbb{E}$. 
Let $\chi_{n}$ be a $C_{0}^{\infty}$ function such that

$$
\begin{aligned}
\chi_{n}(X) & =1 \quad l_{n-1}^{\prime} \leqq|X| \leqq l_{n}^{\prime \prime} \\
& =0 \quad|X| \leqq l_{n-1}^{\prime}-1 \quad \text { or } \quad|X| \geqq l_{n}^{\prime \prime}+1
\end{aligned}
$$

with derivatives bounded uniformly in $n$. We have by the composition formula,

$$
\begin{aligned}
& {\left[\partial_{z} E_{-+} \#_{h} H_{n-1}^{-1} \#_{h} \Delta_{n} \#_{h} H_{n}^{-1}\right](X) } \\
&=\left(\frac{1}{2 \pi h}\right)^{4} \int e^{l Q_{4}(Y) / h}\left[\partial_{z} E_{-+}\left(X-Y_{1}\right) H_{n-1}^{-1}\left(X-Y_{2}\right)\right. \\
&\left.\times\left\{\chi_{n} \Delta_{n}\right\}\left(X-Y_{3}\right) H_{n}^{-1}\left(X-Y_{4}\right)\right] d Y \\
&+\left(\frac{1}{2 \pi h}\right)^{4} \int e^{l Q_{4}(Y) / h}\left[\partial_{z} E_{-+}\left(X-Y_{1}\right) H_{n-1}^{-1}\left(X-Y_{2}\right)\right. \\
&\left.\times\left\{\left(1-\chi_{n}\right) \Delta_{n}\right\}\left(X-Y_{3}\right) H_{n}^{-1}\left(X-Y_{4}\right)\right] d Y .
\end{aligned}
$$

The second term of the R.H.S. is of order $\mathbf{O}\left(h^{\infty}\right)$ by using (4.2). To estimate the first term, we do integration by parts $m+9$ times. We have

$$
\begin{aligned}
\int e^{i Q_{4}(Y) / h}\left[\partial_{z} E_{-+}\left(X-Y_{1}\right) H_{n-1}^{-1}\left(X-Y_{2}\right)\left\{\chi_{n} \Delta_{n}\right\}\left(X-Y_{3}\right) H_{n}^{-1}\left(X-Y_{4}\right)\right] d Y \\
=\int e^{i Q_{4}(Y) / h} L^{m+9}\left[\partial_{z} E_{-+}\left(X-Y_{1}\right) H_{n-1}^{-1}\left(X-Y_{2}\right)\right. \\
\left.\quad \times\left\{\chi_{n} \Delta_{n}\right\}\left(X-Y_{3}\right) H_{n}^{-1}\left(X-Y_{4}\right)\right] d Y .
\end{aligned}
$$

Using (3.13), (3.14), (4.3) and the fact that $|X| \leqq 1 / 2$, we have

$$
\begin{aligned}
& \left|L^{m+9}\left[\partial_{z} E_{-+}\left(X-Y_{1}\right) H_{n-1}^{-1}\left(X-Y_{2}\right)\left\{\chi_{n} \Delta_{n}\right\}\left(X-Y_{3}\right) H_{n}^{-1}\left(X-Y_{4}\right)\right]\right| \\
& \leqq \\
& \quad \sum_{|\beta| \leqq m+9}\left(\frac{1+\|Y\|}{h^{1 / 2}}\right)^{-2(m+9)+|\beta|} h^{|\beta| / 2} \max \left(1,\left(\frac{h}{(\theta+|\operatorname{Im} z|)^{2}}\right)^{3}\right)^{2} \\
& \quad \times(\theta+|\operatorname{Im} z|)^{-1-|\beta|} .
\end{aligned}
$$

On $\Gamma\left(\theta_{n}\right)$, using the scaling relations: $\theta=\theta_{n}=1 / l_{n}$ and $|Y| \geqq\left|Y_{3}\right| \geqq l_{n} / c(c>0)$, we have

$$
\begin{aligned}
\text { L.H.S. } & \leqq C \frac{h^{m+9}}{(1+\|Y\|)^{9}} \max \left(1,\left(h l_{n}^{2}\right)^{3}\right)^{2} \sum_{|\beta| \leqq m+9} l_{n}^{-2 m+2|\beta|-7} \\
& \leqq C \frac{h^{m+9}}{(1+\|Y\|)^{9}} \max \left(1,\left(h l_{n}^{2}\right)^{3}\right)^{2} l_{n}^{11}
\end{aligned}
$$

Since $l_{n} \leqq C h^{-N}$, we see that for $m>23 N-9$, the R.H.S. is small. Using the estimate in (4.5), we have

$$
\left|\left[\partial_{z} E_{-+} \#_{h} H_{n-1}^{-1} \#_{h} \Delta_{n} \#_{h} H_{n}^{-1}\right](X)\right| \leqq C_{M} h^{M}
$$

on $\Gamma\left(\theta_{n}\right)$ for all $M$ and $n \leqq N_{0}$. 


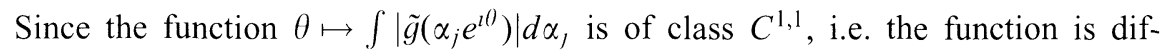
ferentiable and whose derivative is Lipshitz, we have

$$
\int_{\Gamma\left(\theta_{n}\right)}|\tilde{g}(a)| d a=\int \prod_{, \in \Lambda_{n}}\left|\tilde{g}\left(\alpha_{j} e^{i \theta_{n}}\right)\right| d \alpha_{j} \leqq\left(1+C^{\prime} \theta_{n}^{2}\right)^{l_{n}^{2}} \leqq C,
$$

where we used the fact that $\theta_{n}=1 / l_{n}$. We have finally

$$
\sum_{n=1}^{N_{0}} \int_{\Gamma\left(0_{n}\right)}\left|\partial_{z} E_{-+} \#_{h} H_{n-1}^{-1} \#_{h} \Delta_{n} \#_{h} H_{n}^{-1} \tilde{g}(a)\right| d s \leqq C_{M} h^{M}
$$

for all $z$ satisfying $|z| \leqq h^{-1}$ with $|\operatorname{Re} z|>1 / C$ and $|\operatorname{Im} z|>h^{N} / C$ and we used the fact that $N_{0} \leqq$ const $N \log h$.

We are now left with the task of estimating the volume integral $\int_{\Omega\left(\theta_{n}\right)}$. Expanding the wedge product, we have

$$
\begin{aligned}
\mid \int_{\Omega\left(\theta_{n}\right)} & \sum_{j=1}^{\left|\Lambda_{n}\right|} \frac{\partial \tilde{\mathscr{G}}}{\partial \bar{a}_{j}}\left(\partial_{z} E_{-+} \#_{h} H_{n-1}^{-1} \#_{h} \Delta_{n} \#_{h} H_{n}^{-1}\right) d \bar{a}_{l} \wedge d a \mid \\
\leqq & C_{\bar{N}} \int_{0}^{\theta_{n}} \int_{\mathbb{R}\left|\Lambda_{n}\right|} \sum_{j=1}^{\left|\Lambda_{n}\right|} \theta^{\bar{N}} \prod_{l \neq j}\left|\tilde{g}\left(\alpha_{l} e^{l)}\right)\right|\left|\left(\partial_{z} E_{-+} \#_{h} H_{n-1}^{-1} \#_{h} \Delta_{n} \#_{h} H_{n}^{-1}\right)\right| \\
& \times d \bar{a}_{j} \wedge d a_{l} \wedge \cdots \wedge d a_{\left|\Lambda_{n}\right|},
\end{aligned}
$$

where $\bar{N}$ is an integer yet to be determined. We write

$$
\begin{aligned}
& d a_{j}=e^{l \theta} d \alpha_{j}+i e^{l()} \alpha_{j} d \theta, \\
& d \bar{a}_{j}=e^{-l \theta} d \alpha_{j}-i e^{-i \theta} \alpha_{j} d \theta .
\end{aligned}
$$

From (4.4), we have

$$
\left|\partial_{z} E_{-+} \#_{h} H_{n-1}^{-1} \#_{h} \Delta_{n} \#_{h} H_{n}^{-1}\right| \leqq C_{m} \frac{h^{m+9}}{l_{n}^{m}} \frac{1}{\theta^{23+m}}
$$

for all $m$ positive integers. Hence

$$
\begin{aligned}
& \left|\int_{S\left(\theta_{n}\right)} \sum_{j=1}^{\Lambda_{n}} \frac{\partial \tilde{\mathscr{G}}}{\partial \bar{a}_{j}}\left(\partial_{z} E_{-+} \#_{h} H_{n-1}^{-1} \#_{h} \Delta_{n} \#_{h} H_{n}^{-1}\right) d \bar{a}_{j} \wedge d a\right| \\
& \quad \leqq C_{m} C_{\bar{N}}\left|\Lambda_{n}\right| \frac{h^{m+9}}{l_{n}^{m}} \int_{0}^{0_{n}} \theta^{\bar{N}-23-m} \int\left(\prod_{j \neq 1} \tilde{g}\left(\alpha_{j} e^{l(\theta)}\right)\right)\left(\prod_{j \in\left|\Lambda_{n}\right|} d \alpha_{j}\right) d \theta,
\end{aligned}
$$

where the factor $\left|\Lambda_{n}\right|$ comes from summing over $j$. Let $\bar{N}>23+m$, then

$$
\text { R.H.S. } \leqq C_{m} C_{\bar{N}} \frac{\theta_{n}^{\bar{N}-23-m}}{l_{n}^{m-4}} h^{m+9}\left(1+C \theta_{n}^{2}\right)^{l_{n}^{2}} \leqq C_{N} h^{N}
$$

for all $N$. 

lemma

Since $\left\|E_{-+}^{-1}(z)\right\| \leqq C /|\operatorname{Im} z|$, with $C$ independent of $\omega$, we have from Beals'

$$
\left|\partial_{x}^{\alpha} \partial_{\xi}^{\beta} E_{-+}^{-1}\right| \leqq C_{\alpha, \beta}|\operatorname{Im} z|^{-7+\alpha+\beta} \leqq C_{\alpha, \beta} h^{-N(7+\alpha+\beta)},
$$

with $C_{\chi, \beta}$ independent of $\omega$. Hence by the same reasoning we obtain

$$
\left|\left\langle\partial_{z} E_{-+} \#_{h} H_{N_{0}}^{-1} \#_{h} \tilde{\Delta}_{N_{0}+1} \#_{h} E_{-+}^{-1}\right\rangle_{c}\right| \leqq C_{N} h^{N}
$$

for all $N$.

Similarly we obtain, after averaging, that the first (dominant) term in the R.H.S. of (4.1) is bounded:

$$
\left|\left\langle\partial_{z} E_{-+} \#_{h} H_{0}^{-1}\right\rangle_{c}\right| \leqq C
$$

Hence we have

$$
\left|\left\langle\partial_{z} E_{-+} \sharp_{h} E_{-+}^{-1}\right\rangle\right| \leqq C
$$

by summing the series. Clearly by the same argument we have

$$
\left|\partial_{x}^{\alpha} \partial_{\breve{c}}^{\beta}\left\langle\partial_{z} E_{-+} \sharp_{h} E_{-+}^{-1}\right\rangle\right| \leqq C_{\alpha, \beta} .
$$

This proves that $\left\langle\partial_{z} E_{-+} \#_{h} E_{-+}^{-1}\right\rangle$ is a classical symbol in class $S^{0}$.

Proposition 4 yields:

Corollary. For all $N_{0} \in \mathbb{N}, c>0$; there exists $h_{0}$ such that for all $\left.\left.h \in\right] 0, h_{0}\right]$, if $f$ is a $C_{0}^{\infty}$ function, with support contained in $(-B,-c) \cup(c, B)$ satisfying $\left|\partial^{\jmath} f\right| \leqq C_{J} h^{-N_{0 j}}$, then

$$
A_{f}=\int \partial_{\bar{z}} \hat{f}\left\langle\partial_{z} E_{-+}^{(n)} \#_{h} E_{-+}^{-1(n)}\right\rangle d \bar{z} \wedge d z
$$

is a classical symbol in $S_{0}$.

Proof. We construct $\tilde{f}$ such that $\bar{\partial}_{\bar{z}} \tilde{f} \leqq C_{M} h^{-N_{0} M}|\operatorname{Im} z|^{M}$ for all $M \geqq 0$. If $|\operatorname{Im} z| \geqq h^{N^{\prime}} / C$, for some $N^{\prime} \geqq 0$, then we take $M=0$ and apply Proposition 4 . If $|\operatorname{Im} z|<h^{N^{\prime}} / C$, then from Beals' lemma, we have

$$
\left|\partial_{x}^{\alpha} \partial_{\xi}^{\beta}\left\langle\partial_{z} E_{-+} \#_{h} E_{-+}^{-1}\right\rangle\right| \leqq C_{\alpha, \beta} \frac{1}{|\operatorname{Im} z|^{7+\alpha+\beta}} .
$$

Hence

$$
\begin{aligned}
\left|\partial_{z} \tilde{f} \partial_{x}^{\alpha} \partial_{\varepsilon}^{\beta}\left\langle\partial_{z} E_{-+} \#_{h} E_{-+}^{-1}\right\rangle\right| & \leqq C_{\alpha, \beta, M} h^{-N_{0} M}|\operatorname{Im} z|^{M-\alpha-\beta-7} \\
& \leqq C_{\alpha, \beta, M} h^{-N_{0} M} h^{(M-\alpha-\beta-7) N^{\prime}} .
\end{aligned}
$$

By choosing $N^{\prime}=2 N_{0}$, we have

$$
\left|\partial_{z} \tilde{f} \partial_{x}^{\alpha} \partial_{\xi}^{\beta}\left\langle\partial_{z} E_{-+} \#_{h} E_{-+}^{-1}\right\rangle\right| \leqq C_{\alpha, \beta, M} h^{N_{0}(M-2 \gamma-2 \beta-14)} .
$$

Clearly, if $2(\alpha+\beta) \leqq M-14$, we have a well defined p.d.o.. Hence with the condition $\left|\partial^{j} f\right| \leqq C_{j} h^{-N_{0} l}, A_{f}$ is a classical symbol in the class $S_{0}$. 


\section{Conclusion}

We now state our main theorems. (Recall that $\operatorname{supp} g \subset(-p, p)$ and range $v \subset$ $[-1,1]$.)

Theorem 1. There exist $\rho_{l}^{(n)}(t)(n \in \mathbb{N})$ in $C_{0}^{\infty}(\mathbb{R} \backslash\{0\})$ with support contained in $(-p, p)$ such that for all $N_{0} \in \mathbb{N}, c>0$, there exists $B_{0}$, such that if $f \in$ $C_{0}^{\infty}((-B,-c) \cup(c, B)+(2 n+1) B)$ satisfies $\left|\partial^{\prime} f\right|=\underline{O}\left(B^{N_{0} J}\right)$ with $B \geqq B_{0}>0$, then for every $m \in \mathbb{N}$ :

$$
\begin{aligned}
\left\langle\tilde{\operatorname{Tr}} f\left(P_{B, V}^{(I)}\right)\right\rangle= & \int f(E) d \rho_{B}(E) \\
= & B \int f(t+(2 n+1) B) \rho_{0}^{(n)}(t) d t \\
& +\int f(t+(2 n+1) B) \rho_{1}^{(n)}(t) d t \\
& +B^{-1} \int f(t+(2 n+1) B) \rho_{2}^{(n)}(t) d t+\cdots \\
& +B^{-(m-1)} \int f(t+(2 n+1) B) \rho_{m}^{(n)}(t) d t+\mathbf{O}\left(B^{-m}\right),
\end{aligned}
$$

where $d \rho_{B}(E)$ is the "non-random" density of states measure. One has for example:

$$
\begin{aligned}
& \rho_{0}^{(n)}(t)=\frac{1}{2 \pi} \int \prod_{l} g\left(\alpha_{l}\right) d \alpha_{l} \int_{t_{t}} \frac{d s}{\|\nabla V\|}, \\
& \rho_{1}^{(n)}(t)=\frac{2 n+1}{8 \pi} \int \prod_{l} g\left(\alpha_{l}\right) d \alpha_{l} \frac{d}{d t}\left(\int_{t_{t}} \frac{\Delta V}{\|\nabla V\|} d s\right),
\end{aligned}
$$

where $\mathscr{V}_{t}$ is the intersection of the curve $V(x, \xi)=t$ with the unit square centered at $(0,0)$. Note that $\rho_{0}^{(n)}$ is independent of $n$.

In particular, if $v$ has its support contained in the unit square, then

$$
\rho_{0}^{(n)}=\frac{1}{2 \pi} \int F_{0}(\gamma) g\left(\frac{t}{\gamma}\right) \frac{d \gamma}{|\gamma|}
$$

where

$$
F_{0}(\gamma)=\int_{v=;} \frac{d s}{\|\nabla v\|}
$$

and

$$
\rho_{1}^{(n)}=\frac{2 n+1}{8 \pi} \int F_{1}(\gamma) g\left(\frac{t}{\gamma}\right) \frac{d \gamma}{|\gamma|}
$$

where

$$
F_{1}(\gamma)=\frac{d}{d \gamma}\left(\int_{v=\gamma} \frac{\Delta v}{\|\nabla v\|} d s\right) .
$$

Proof. Direct consequence of Proposition 4 and its corollary and straightforward computation by using the asymptotic expansion for $E_{-+}^{-1}$ and $\partial_{z} E_{-+}$.

Remark. Because of the extra integration over the random variable $\alpha$, when we compute $d \rho_{m} / d t$, we somehow are always computing $\partial g / \partial \alpha$. Since $g$ is a $C_{0}^{\infty}$ function, so is $\rho_{m}$. 
If we further assume that $v \geqq 0$ and that the support of $v_{l}$ intersect so that $\sum_{l \in \mathbb{Z}^{2}} v_{i} \geqq s>0$, then we have:

Theorem 2. There exist $\rho_{l}^{(n)}(t)(n \in \mathbb{N})$ in $C_{0}^{\infty}(-p, p)$ such that for all $N_{0} \in \mathbb{N}$, $c>0$, there exists $B_{0}$, such that if $f \in C_{0}^{\infty}((-B, B)+(2 n+1) B)$ satisfies $\left|\partial^{j} f\right|=$ $\mathbf{O}\left(B^{N_{0 J}}\right)$ with $B \geqq B_{0}>0$, then for every $m \in \mathbb{N}$ the expansion (5.1) holds.

Proof. We only need to prove that the expansion (5.1) holds for $f \in C_{0}^{\infty}((-c, c)+$ $(2 n+1) B)$ for some $c>0$ to be determined. We use a similar construction to the one used in the proof of Proposition 4 in Sect. IV. We take $H_{n}$ to be the reduced operator corresponding to the potential

$$
V_{n}(\bar{x})=\sum_{i \in \Lambda_{n}} \alpha_{l} v(\bar{x}-i)+\sum_{l \in \mathbb{Z}^{2} \backslash \Lambda_{n}} v(\bar{x}-i) .
$$

Instead of complex dilating in $\alpha$ as in the proof of Proposition 4 , we complex translate in $\alpha$ :

$$
\alpha_{J} \mapsto \alpha_{J}-i(\operatorname{sign} \operatorname{Im} z) \delta_{n},
$$

where $\delta_{n}=1 / l_{n}$. We assume that $\operatorname{Im} z<0$ (the other case can be treated in the same way). Let $H_{n}(\delta)$ be the reduced operator corresponding to $\alpha_{j}$ replaced by $\alpha_{j}+i \delta\left(0<\delta \leqq \delta_{n}\right)$. The principal symbol for $h^{-1} H_{n}(\delta)$ is

$$
V_{n}^{\delta}(x, \xi)-z=\sum_{j \in \Lambda_{n}} \alpha_{j} v_{j}(x, \xi)+i \delta \sum_{j \in \Lambda_{n}} v_{j}(x, \xi)+\sum_{j \in \mathbb{Z}^{2} \backslash \Lambda_{n}} v_{j}(x, \xi)-z
$$

Let $\phi \in L^{2}(\mathbb{R})$; we have

$$
\begin{aligned}
\operatorname{Re}\left\langle\phi,\left(V_{n}^{\partial}-z\right) \phi\right\rangle & =\left\langle\phi, \sum_{j \in \Lambda_{n}} \alpha_{j} v_{j} \phi\right\rangle+\left\langle\phi, \sum_{j \in \mathbb{Z}^{2} \backslash \Lambda_{n}} v_{j} \phi\right\rangle-\operatorname{Re} z\langle\phi, \phi\rangle, \\
\operatorname{Im}\left\langle\phi,\left(V_{n}^{\partial}-z\right) \phi\right\rangle & =\delta\left\langle\phi, \sum_{j \in \Lambda_{n}} v_{j} \phi\right\rangle+|\operatorname{Im} z|\langle\phi, \phi\rangle \\
& \geqq \delta\left\langle\phi, \sum_{j \in \Lambda_{n}} v_{j} \phi\right\rangle \geqq 0 .
\end{aligned}
$$

Since $\sum_{\jmath \in \mathbb{Z}^{2}} v_{j} \geqq s>0$, we certainly have, for $h$ small enough

$$
\left\langle\phi, \sum_{j \in \mathbb{Z}^{2}} v, \phi\right\rangle>s / 2\langle\phi, \phi\rangle
$$

Let $\tilde{\alpha}=\max \left(|\alpha|_{\infty}, 3\right)$. For $\phi$ such that $\left\langle\phi, \sum_{\jmath \in \Lambda_{n}} v_{j} \phi\right\rangle \leqq s /\left(\tilde{\alpha}^{2}\right)\langle\phi, \phi\rangle$, we have

$$
\left|\left\langle\phi, \sum_{j \in \Lambda_{n}} \alpha_{j} v, \phi\right\rangle\right| \leqq \frac{s}{\tilde{\alpha}}\langle\phi, \phi\rangle .
$$

We also have from (5.2)

$$
\left\langle\phi, \sum_{j \in \mathbb{Z}^{2} \backslash \Lambda_{n}} v, \phi\right\rangle \geqq s\left(\frac{1}{2}-\frac{1}{\tilde{\alpha}^{2}}\right)\langle\phi, \phi\rangle .
$$


Hence

$$
\operatorname{Re}\left\langle\phi,\left(V_{n}^{\delta}-z\right) \phi\right\rangle \geqq s\left(\frac{1}{2}-\frac{1}{\tilde{\alpha}^{2}}\right)\langle\phi, \phi\rangle-\operatorname{Re} z\langle\phi, \phi\rangle .
$$

Taking $c=s / 19$, we have $|\operatorname{Re} z| \leqq s / 19$. Therefore

$$
\max \left(\left|\operatorname{Re}\left\langle\phi,\left(V_{n}^{\delta}-z\right) \phi\right\rangle\right|,\left|\operatorname{Im}\left\langle\phi,\left(V_{n}^{\delta}-z\right) \phi\right\rangle\right|\right) \geqq \frac{\delta s}{\tilde{\alpha}^{2}}\langle\phi, \phi\rangle .
$$

Let $h S_{\delta, z}=h^{-1} H_{n}(\delta)-V_{n}^{\partial}+z$. The symbols $\partial_{\delta} S, \partial_{z} S$ belong to $S^{0}$. Hence for $h$ sufficiently small, we have

$$
\operatorname{dist}\left(0, \mathscr{N} \mathscr{R}_{H_{n}(\delta)}\right) \geqq c^{\prime} h(\delta+|\operatorname{Im} z|),
$$

where $\mathscr{N} \mathscr{R}_{H_{n}(\partial)}$ is the numerical range of $H_{n}(\delta)$. We therefore have

$$
\left\|H_{n}^{-1}(\delta)\right\| \leqq \frac{1}{c^{\prime} h(\delta+|\operatorname{Im} z|)}
$$

The rest of the proof follows exactly that of Theorem 1, with $\delta$ replacing $\theta$.

\section{References}

[Bea] Beals, R.: Characterization of pseudo-differential operators and applications. Duke Math. J. 44 (1977)

[Bel] Bellissard, J.: Cours a l'École normale supérieure. (1992-1993)

[BGI] Brézin, E., Gross, D., Itzykson, C.: Density of states in the presence of a strong magnetic field and random impurities. Nucl. Phys. B 235 (1984)

[CFKS] Cycon, H.L., Froese, R.G., Kirsch, W., Simon, B.: Schrödinger Operators. Lecture Notes in Physics, Berlin-Heidelberg-New york: Springer Verlag, 1987

[HS] Helffer, B., Sjöstrand, J.: Equation de Schrödinger avec champ magnétique et équation de Harper. Lecture Notes in Physics 345, 1989

[KP] Klein, A., Perez, J.-F.: On the Density of states for random potentials in the presence of a uniform magnetic field. Nucl. Phys. B251 (1985)

[Pa] Pastur, L.: Spectral properties of disordered systems in one-body approximation. Commun. Math. Phys. 75 (1980)

[S] Sjöstrand, J.: Microlocal analysis for the periodic magnetic Schrödinger equation and related questions. Lecture Notes in Math. 1495, 1989

[We] Wegner, F.: Exact density of states for lowest Landau level in white noise potentialsuperfield representation for interactive systems. Zeitschrift Physik B-Condensed Matter $51(1993)$

Communicated by B. Simon 
